\title{
Integration of Olfactory Information in a Spatial Representation Enabling Accurate Arm Choice in the Radial Arm Maze
}

\author{
Pierre Lavenex ${ }^{1}$ and Françoise Schenk \\ Institut de Physiologie \\ Université de Lausanne \\ CH-1005 Lausanne, Switzerland
}

\begin{abstract}
The aim of the present study was to determine whether and how rats can use local olfactory cues for spatial orientation. Rats were trained in an eight-arm radial maze under different conditions as defined by the presence or absence of supplementary olfactory cues marking each arm, the availability of distant visuospatial information, and the illumination of the maze (light or darkness). The different visual conditions were designed to dissociate among the effects of light per se and those of visuospatial cues, on the use of olfactory cues for accurate arm choice. Different procedures with modifications of the arrangement of olfactory cues were used to determine if rats formed a representation of the spatial configuration of the olfactory cues and if they could rely on such a representation for accurate arm choice in the radial maze. The present study demonstrated that the use of olfactory cues to direct arm choice in the radial arm maze was critically dependent on the illumination conditions and implied two different modes of processing of olfactory information according to the presence or the absence of light. Olfactory cues were used in an explicit manner and enabled accurate arm choice only in the absence of light. Rats, however, had an implicit memory of the location of the olfactory cues and formed a representation of the
\end{abstract}

\footnotetext{
${ }^{1}$ Corresponding author.
}

spatial position of these cues, whatever the lighting conditions. They did not memorize the spatial configuration of the olfactory cues per se but needed these cues to be linked to the external spatial frame of reference.

\section{Introduction}

Numerous studies of spatial orientation in rats have been designed to show either that a specific type of sensory information is not sufficient to enable a rat to solve a particular task or, in contrast, that a specific type of sensory information is the necessary information enabling a rat to solve a particular task. Very few studies, however, have attempted to analyze the interactions between the different types of sensory information and how they could be used at different stages of learning or according to the specific demands of the task (for exceptions, see Etienne et al. 1990; Alyan and Jander 1994).

Most studies concerned with the processing of spatial information consider only visual cues and do not evaluate the role that olfactory cues might play. Spatial information derived from distant visual cues appears to predominate over other types of information in many different spatial tasks (e.g., Suzuki et al. 1980; Lavenex and Schenk 1995). There are also evidences showing that proximal visual cues are incorporated in a spatial representation (see Poucet 1993). Olfactory cues, however, are considered to interfere with the study of the visual information used to solve spatial tasks. In general, olfactory cues are suppressed or made irrelevant in studies concerned with the processing of visuospatial information.

LEARNING \& MEMORY 2:299-319 @ 1995 by Cold Spring Harbor Laboratory Press ISSN1072-0502/96 \$5.00

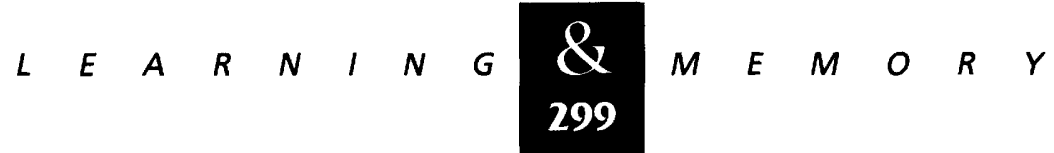


Thus, little is known about the information that rats might derive from olfactory cues for spatial orientation. This is surprising, as rats are known for their well-developed abilities to process olfactory information (e.g., Staubli et al. 1987; Youngentob et al. 1990; Slotnick et al. 1991; Lu et al. 1993). Previous experiments concerned with the use of olfactory cues in the eight-arm radial maze demonstrated that even though intramaze olfactory cues could contribute to the complex sensory information enabling accurate performance (Buresova and Bures 1981), these cues failed to direct arm choice (Olton and Samuelson 1976; Olton and Collison 1979) and were not critical for accurate arm choice (Zoladek and Roberts 1978). Nevertheless, it had been suggested that olfactory cues might be used if visuospatial cues were made less salient or even suppressed ( $\mathrm{Zo}$ ladek and Roberts 1978; Olton and Collison 1979).

In general, olfactory cues appeared to act more as contextual cues, rather than as cues enabling arm choice. Adding a new odor into the experimental design induced changes in the general behavior of the rat, without modifying its accuracy (Olton and Samuelson 1976). Similarly, experiments on spontaneous alternation showed that even though the odor did not enable arm choice, changing the maze odor modified the spontaneous alternation rate (Still and Macmillan 1975).

Although olfactory information does not seem to play a dominant role, it is certainly not neglected. The question is, thus, to determine how olfactory information is linked to information provided by other sensory modalities. A previous study has shown that hamsters derived spatial information from olfactory cues associated to particular locations defined by distant visual cues (Tomlinson and Johnston 1991). No study, however, has shown that animals elaborate a representation of the spatial configuration of the olfactory cues independently of any visual information. Moreover, no study has shown that olfactory cues could be integrated in a representation of the spatial configuration of these cues and that rats could rely on this representation for accurate orientation. Nevertheless, the idea of a cognitive map built upon olfactory cues has been already proposed by Baker (1978). His theoretical model proposed that windborn odors could allow the establishment of a map of the environment based on olfactory cues. The limitation of this model is that the map would only be effective if a wind is blowing and could only give a directional reference. Pigeons are supposed to rely on such an olfactory map based on windborn odors in order to home over long distances from unfamiliar areas. The experimental data support this hypothesis, but the nature of this map is still under debate (see Papi 1990).

The aim of the present study is to determine how rats can use local environmental olfactory cues for spatial orientation. We wanted to determine whether rats formed a representation of the spatial configuration of these cues, which they could use for accurate orientation, as is the case with visual cues.

Rats were trained in a classical eight-arm radial maze task design. Different conditions were designed to determine the influence of visual information on the use of olfactory cues for accurate arm choice. They were defined by the presence or absence of supplementary olfactory cues marking each arm, the availability of distant visual cues, and the illumination of the maze (light or darkness). A special procedure with modified arrangements of the olfactory cues was used to determine if rats had formed a representation of the spatial configuration of the olfactory cues. This procedure has been used previously in different apparatus to study the spatial information derived from environmental cues (Suzuki et al. 1980; Poucet et al. 1986; Tomlinson and Johnston 1991). Rats were first trained with olfactory cues remaining stable within the maze. Then the configuration of the olfactory cues was rearranged to determine if rats had formed a representation of the configuration of the olfactory cues. Other experiments with an interruption procedure were also realized to verify that rats can actually rely on such a representation for accurate arm choice in the radial maze.

\section{Experiment 1}

This experiment was designed to examine, first, under which conditions rat use intramaze olfactory cues for accurate arm choice in the eightarm radial maze and, second, whether they memorize the location of olfactory cues. Rats were trained on an elimination task in which they had to visit the eight arms of the maze. Six different experimental conditions were designed according to the presence or absence of supplementary olfactory cues, the availability of distant visuospatial cues, and the illumination conditions of the maze (light or darkness).

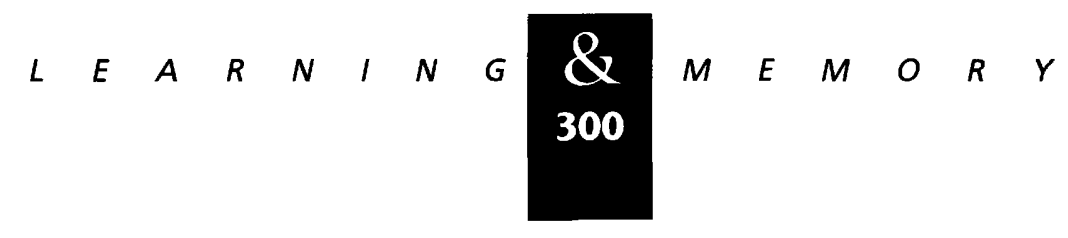




\section{Material and Methods}

\section{SUBJECTS}

Fifty-three male, Long-Evans hooded rats were used as subjects. They were 4 to 5 months old at the beginning of the experiment. Some of the rats had been tested previously in the Morris maze in an open-field arena, but none had ever been tested in a radial maze or in the experimental room used in this study. The animals were housed in groups of three or four individuals in glass cages $(40 \times 40 \times 50 \mathrm{~cm})$ containing large wooden nest boxes or individually in Plexiglas cages $(22.5 \times 36 \times 18 \mathrm{~cm})$. Throughout the testing period they had water ad libitum, but the distribution of food was limited to maintain the animals at $95 \%$ of their free-feeding weight.

\section{APPARATUS}

The apparatus was an eight-arm radial maze made of transparent Plexiglas tunnels (12×12×60 $\mathrm{cm})$ arranged on wooden supports $(12 \times 60 \mathrm{~cm})$ to form a transparent enclosed radial maze, placed on a large rotating board. The central choice area was $33 \mathrm{~cm}$ in diameter and was covered by a Plexiglas lid. The floor of the arms and of the central choice area was covered by a plastic sheet. The arms and the central platform were separate from each other and could be moved independently. Before each test, arms were baited with a drop of diluted condensed milk placed on a small glass plate at the end of each arm.

The experiments were conducted in a $2.5 \times 4$ $m$ room containing a heterogeneous collection of extramaze stimuli (table, sink, blackboard, etc.). Different groups were designed according to the access to distant visuospatial cues, the illumination of the maze (light or darkness), and the presence of supplementary olfactory cues. In the white-light illumination condition, the maze was indirectly lit by two $60-\mathrm{W}$ light bulbs directed toward the ceiling. The deprivation of visual cues was realized either by using nontransparent (translucent) tunnels or by testing in darkness (infrared illumination). The use of translucent tunnels prevented rats from seeing anything outside the maze but left the inside of the maze lighted. In the darkness condition, the maze was lit by an infrared projector (Infralite IR-RS 4060: narrow-band light emission, wavelength $880 \mathrm{~nm}$ ), which provided light outside the visible spectrum. An infrared viewer
(Find-R-Scope 85100, Cotraco establishment) was used to enable experimental manipulation and direct observation of the animals. An infrared camera was placed above the center of the maze. All the tests were video recorded to ensure accurate collection of the data.

Supplementary olfactory cues could be added to each arm according to the experimental group. These cues were made of eight different alimentary aromas (McCormick) diluted with water (1: $10)$ and deposited on blotting paper affixed to the tunnel's roof. Bands of blotting paper $(3 \times 60 \mathrm{~cm})$ were fixed on the full length of the tunnel's roof (i.e., from the entrance to the distal end of the tunnel) and received $0.5 \mathrm{ml}$ of the diluted aroma. Five minutes elapsed between the deposit of the olfactory cues and the run of the first rat. The olfactory cues were reapplied after four rats had performed the task and, again, 5 min elapsed until the next rat was placed in the maze.

\section{PROCEDURE}

Rats were assigned to six different experimental conditions designed according to the availability of distant visuospatial cues, the illumination of the maze, and the presence of supplementary olfactory cues (Fig. 1): transparent + odor $(n=8)$, transparent $(\mathrm{n}=6)$, translucent + odor $(n=7)$, translucent $(n=7)$, darkness + odor $(n=9)$, and darkness $(n=9)$. A seventh group of rats $(n=7)$ was tested with translucent tunnels and supplementary olfactory cues, but all manipulations when rats were outside the maze (handling of rats, manipulation of maze arms, etc.) were made under infrared light illumination, as in the darkness condition. This ensured that rats had no access to any distant visuospatial cues from the room even when they were not inside the maze. This contrasts with the first condition with translucent tunnels and supplementary olfactory cues, in which rats had access to distant visuospatial cues when we placed them in or removed them from the maze. Animals from all conditions were given one trial session daily according to the following procedure.

STABLE CONFIGURATION OF THE MAZE ARMS

Rats were first tested with a free-choice procedure for 13 days. The rat was placed in a plastic cylinder at the center of the arena always from the same position in the experimental room. The rat

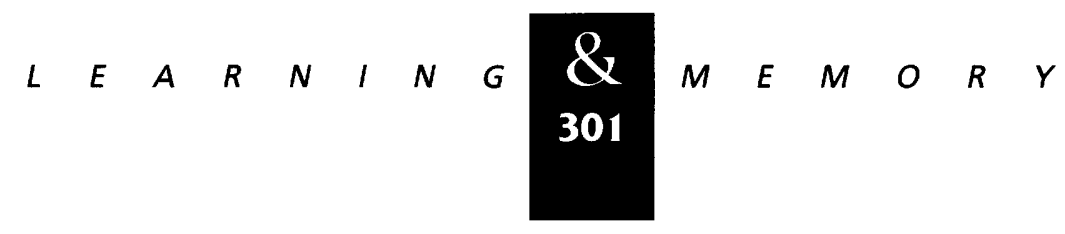




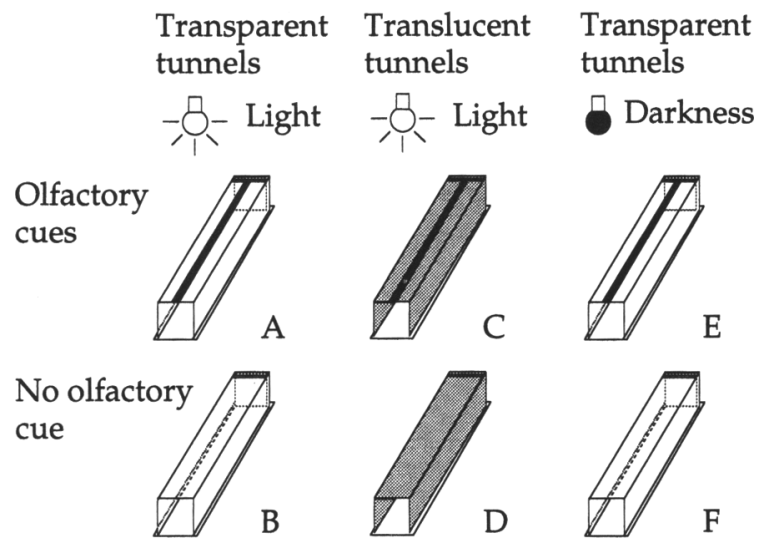

Figure 1: Schematic representation of the $3 \times 2$ experimental design with different conditions according to the three different visual and two olfactory conditions: $(A)$ transparent + odor (visuospatial cues accessible, olfactory cues present); $(B)$ transparent (visuospatial cues accessible, no olfactory cue); $(C)$ translucent + odor (no visuospatial cues but maze lighted, olfactory cues present); (D) translucent (no visuospatial cues but maze lighted, no olfactory cue); (E) darkness + odor (no light, olfactory cues present); $(F)$ darkness (no light, no olfactory cue).

could move freely inside the cylinder. After a few seconds, the cylinder was raised and the rat was allowed to choose arms. This starting procedure was used to avoid forcing the rat to always face a particular arm when it started visiting the maze. Rats were allowed to patrol the maze until all eight arms had been visited. Then two extra choices were given to avoid interrupting the rat right after the last correct choice. The first day of testing was considered as an exploration session and was not included in the analysis of the results.

REARRANGEMENT OF THE CONFIGURATION OF THE MAZE ARMS

The procedure was the same as with the stable configuration of the maze arms (i.e., the animals were allowed free choice until they had visited the eight arms + two extra choices), but the maze arms were pseudorandomly redistributed each day. This rearrangement left the geometric shape of the maze unaffected (regular eight-arm radial maze), but the configuration of the olfactory cues was reorganized. When the arms of the maze were rearranged, the floor of the arms were also moved. Thus, any possible olfactory trace left on the arm floor was displaced with the olfactory-marked tun- nel. The floor of the central choice area was undisturbed and remained in the same orientation.

This phase lasted 6 days and followed the first 13 days of training with the stable configuration of the maze arms. The three groups of rats trained in the stable configuration of the maze with supplementary olfactory cues (transparent + odor, translucent + odor, darkness + odor) were tested with daily rearranged configurations of the maze. The animals trained in darkness with no supplementary olfactory cues were also tested to assess the role of uncontrolled olfactory traces that the animals may have left in the apparatus.

The performance is described with regards to the number of errors (entries into previously visited arms) and to the strategy used to patrol the maze (angles separating two arms visited successively).

\section{Results}

The results of both groups of rats tested in translucent tunnels + odor (light on or off while the rats were manipulated outside the maze) were similar with regard to the number of errors and to the strategy used to patrol the maze; these two groups have been merged for all of the following analyses.

\section{STABLE CONFIGURATION OF THE MAZE ARMS}

The number of errors made before completing the maze is represented on Figure 2 (blocks 1-4). A two-way ANOVA with repeated measures revealed significant differences between groups $[F(5,47)=3.43, P=0.01]$ and a decrease of the number of errors throughout training for all groups $[F(3,141)=34.29, P=0.0001$; interaction: $F(15,141)=1.24, P=\mathrm{ns}]$.

As expected, rats trained in the absence of visuospatial and supplementary olfactory cues made more errors than rats trained in transparent tunnels. At the end of training (block 4), a twoway ANOVA revealed significant differences between the visual conditions $[F(2,47)=4.03$, $P=0.009$ ], no difference between the olfactory conditions $[F(1,47)=1.59, P=n s]$, but a significant interaction between visual and olfactory conditions $[F(2,40)=3.7, P=0.04]$. In the transparent tunnels conditions, there was no difference between rats trained with or without supplementary

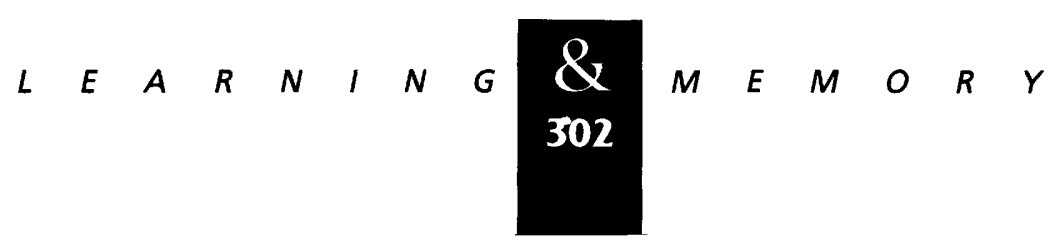


Standard procedure

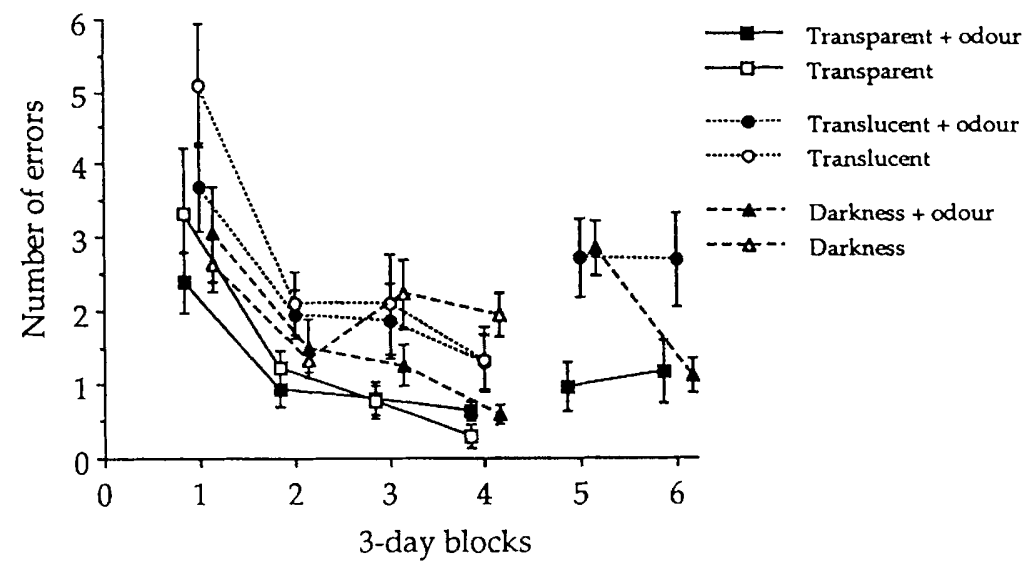

Figure 2: Number of errors per block of 3 days during the two different phases of Experiment 1 . Blocks 1 and 4 were conducted with a stable configuration of maze arms. Blocks 5 and 6 were conducted with a configuration of maze arms rearranged each day. olfactory cues $[F(1,12)=2.85, P=$ ns $]$; the performance was already optimal in the absence of the olfactory cues. In darkness, rats trained in the presence of supplementary olfactory cues made fewer errors than rats trained without these cues $[F(1,16)=17.69, P=0.0007]$ and improved their performance to the level of rats trained in transparent tunnels. In translucent tunnels, the presence of supplementary olfactory cues did not improve performance $[F(1,19)=0.002, P=\mathrm{ns}]$.

The strategy used by rats to patrol the maze depended on the visual conditions and on the presence of supplementary olfactory cues (Fig. 3). The analysis of the number of adjacent visits (angles of $45^{\circ}$ between successively visited arms) shows significant differences between the visual conditions $[F(2,40)=11.21, P=0.0001]$, no difference between the olfactory conditions
$[F(1,40)=0.13, P=n s]$, but a significant interaction between the visual and the olfactory conditions $[F(2,40)=7.85, P 0.002]$. In the transparent tunnels, there was no difference between rats trained in the presence or in the absence of supplementary olfactory cues $[F(1,12)=0.72, P=n s]$. In the translucent conditions, the animals trained without supplementary olfactory cues chose more adjacent arms than those trained in the presence of olfactory cues, though the difference just failed to reach significance $[F(1,19)=3.84, P=0.07]$. In darkness, the animals trained without supplementary olfactory cues chose adjacent arms less often than rats trained in the presence of supplementary olfactory cues $[F(1,16)=10.48, P=0.006]$. The detailed analysis of the other angles between arms visited successively is not presented as it gives similar information.

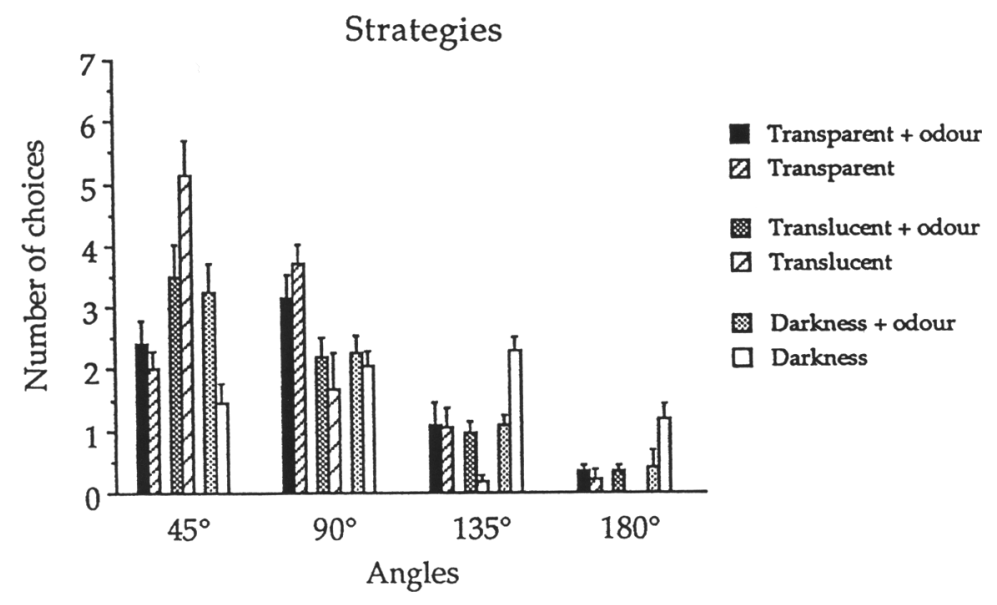

Figure 3: Mean number of different angles separating two successively visited arms in the first eight choices during the last block with a stable configuration of the maze arms (block 4).

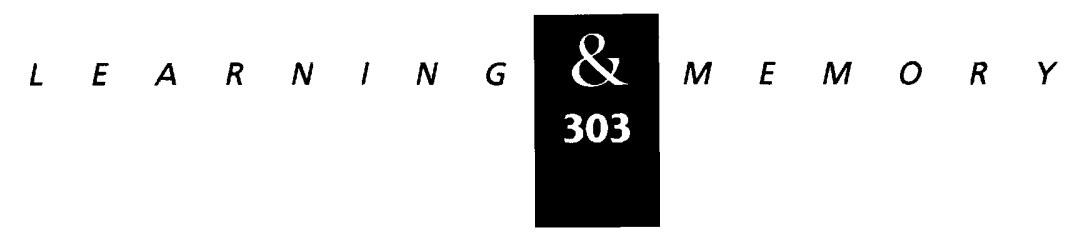




\section{REARRANGEMENT OF THE CONFIGURATION OF} THE MAZE ARMS

A two-way ANOVA with repeated measures conducted on the last block of acquisition with a stable configuration of the olfactory cues (Fig. 2, block 4) and the two blocks with a rearranged configuration of the olfactory cues (Fig. 2, blocks 5 and 6) indicated groups and blocks effects [groups: $\quad F(2,28)=3.84, \quad P=0.04 ; \quad$ blocks: $F(2,56)=10.85, \quad P=0.0001$; $\quad$ interaction: $F(4,56)=2.52, P=0.06]$. An analysis of the performance of each group following the modification of the position of the olfactory cues showed that rats trained in transparent tunnels did not react to the rearrangement of the cues $[F(2,14)=0.79, P=n s]$, whereas rats trained with translucent tunnels made more errors following the rearrangement of the olfactory cues $[F(2,26)=4.08, P=0.03$, block $4<$ block $5=$ block 6]. Rats trained in darkness showed a transitory increase in the number of errors in the first block with rearranged configurations of the olfactory cues. However, in the second block of trials with modified configurations of the olfactory cues, they did not make more errors than in the last block of acquisition with a standard configuration of the olfactory cues $[F=7.35$, $P=0.0025$, block $4=$ block $6<$ block 5). Rats trained in darkness without supplementary olfactory cues also experienced the same rearrangement of the maze arms as controls (not shown). The manipulation of the maze arms without any supplementary olfactory cues did not produce any disruption of performance $[F(2,16)=1.55$, $P=$ ns].

\section{Discussion}

These results indicate that the use of olfactory cues for accurate arm choice in the eight-arm radial maze is critically dependent on the absence of light and not on the absence of distant visuospatial cues, at least during initial learning. Rats benefited from the presence of supplementary olfactory cues only when they were trained in darkness (infrared illumination). Moreover, the efficacy to perform, the reaction to arms' rearrangement, and the strategy to patrol the maze were differently affected by olfactory and visual conditions.

In the presence of distant visuospatial information, we found no effect of the addition of olfactory cues on task efficacy, as rats already exhibited optimal performance in the absence of these cues. Rats trained in darkness with supplementary olfactory cues were able to rely on these cues and exhibited a performance similar to that of the animals allowed access to the distant visuospatial cues. In contrast, the poor performance of rats trained in darkness without supplementary olfactory cues clearly showed that the uncontrolled olfactory traces present on the floor of the apparatus were not sufficient to compensate for the absence of visuospatial cues and to enable accurate arm choice.

In the absence of distant visuospatial information but in a lighted maze (translucent tunnels), rats did not benefit from the presence of supplementary olfactory cues, even though no visual cues were available. Their performance remained lower than that of rats trained in the presence of distant visuospatial information. Rats trained in translucent tunnels but introduced under infrared illumination were tested to verify that the difference observed between rats trained in darkness or in translucent tunnels was not attributable to the fact that one group had access to visuospatial information when outside the maze, whereas the other did not. The results show that the lighting conditions during the manipulation while the rats were outside the maze had no influence on their behavior inside the maze.

The daily rearrangement of the olfactory cues had a different effect in each maze condition. Rats trained under white light in transparent tunnels did not show any disruption of their performance. Rats trained with translucent tunnels showed a persistent disruption of their performance as shown by an increased error rate. Rats trained in darkness showed only a transient increase in error rate in the first block with daily rearrangement of the scented arms. In the second block of trials with rearranged arms, their performance was again similar to that observed when the configuration of the olfactory cues was stable. These results demonstrated that rats trained in darkness had memorized the configuration of the olfactory cues but did not need the location of these cues to remain stable from day to day for accurate arm choice. Rats trained in translucent tunnels had also memorized the location of the olfactory cues, even though they did not rely on these cues for accurate arm choice. It is very likely that rats trained in transparent tunnels had also memorized the location of the olfactory cues but did not react to their rearrangement because of the predominance of the visuospatial information.

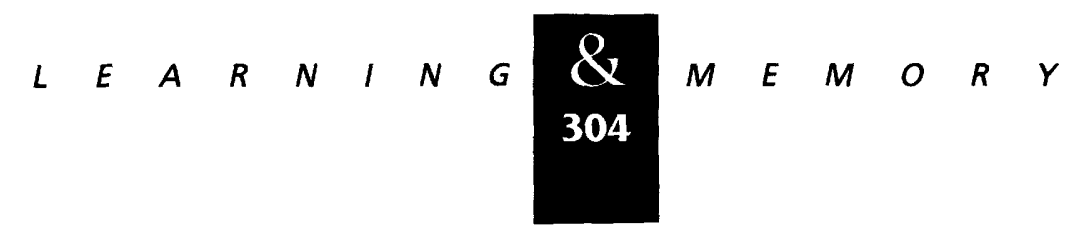


The strategy used by rats to patrol the maze (Fig. 3) depended on the presence of supplementary olfactory cues and on the visual conditions. In the presence of supplementary olfactory cues, the animals of all groups adopted a similar strategy regardless of the visual conditions. They chained preferentially arms separated by $45^{\circ}$ or $90^{\circ}$ and chained arms separated by $135^{\circ}$ or $180^{\circ}$ less frequently. This corresponds to the way of patrolling an eight-arm radial maze usually observed when rats have full access to distant visuospatial cues (this paper; Suzuki et al. 1980; Yoerg and Kamil 1982; Innis and Macgillivray 1987). In the absence of olfactory cues, however, rats used different strategies according to the visual conditions. In the absence of distant visuospatial cues but in a lighted maze (translucent tunnels), rats tended to visit most frequently arms separated by $45^{\circ}$, which corresponds to the chaining of adjacent arms. These results are consistent with other findings showing a tendency for rats to choose more adjacent arms when tested in an enclosed maze with a reduced access to discriminative visuospatial cues (Innis and Macgillivray 1987) or in an impoverished environment (Suzuki et al. 1980). Rats tested in darkness in the absence of supplementary olfactory cues did not exhibit such a tendency. In contrast, they tended to successively visit arms separated by larger angles $\left(135^{\circ}\right.$ of $\left.90^{\circ}\right)$.

The lack of cues enabling the discrimination of the arms may have led the animals to rely more on the path integration mechanism to solve the task. In the absence of vision, chaining arms separated by larger angles could reduce the incremental error generated by each directional change (Schenk et al. 1990). The difference between both groups trained in the absence of visuospatial and supplementary olfactory cues (darkness and translucent tunnels) shows that the presence or the absence of light led rats to use completely different strategies to solve the task. This is coherent with the results showing that rats did not rely on the supplementary olfactory cues when the maze was illuminated, even when visuospatial cues were suppressed.

In conclusion, the results of this experiment reveal three different properties of olfactory cues. First, supplementary olfactory cues enable accurate arms choice only in darkness but not when the maze is lighted. Second, rats are sensitive to the modification of the location of the olfactory cues although distant visuospatial cues are suppressed, which showed that they formed a repre- sentation of the spatial configuration of the olfactory cues. Third, in darkness, rats are capable of accurate arm choice even if the olfactory cues do not stay in a stable configuration from day to day.

\section{Experiment 2}

The results of the first experiment indicate that rats are sensitive to the modification of the position of the supplementary olfactory cues in the absence of distant visuospatial cues. However, the olfactory cues enable accurate arm choice only in darkness. The aim of the second experiment was to determine more specifically whether rats rely on the configuration of the olfactory cues and whether they can use the olfactory cues as a list of independent items marking each arm. We wanted also to determine whether previous training in one experimental condition could modify the rat's reliance on the olfactory or spatial information in other experimental conditions.

\section{Materials and Methods}

SUBJECTS

The subjects were the rats from the groups trained in three different conditions with olfactory cues [transparent + odor $(n=8)$, translucent + odor $(n=7)$, darkness + odor $(n=9)]$ used in the first experiment. The experimental conditions were the same as in Experiment 1.

\section{APPARATUS}

The apparatus was the same as in Experiment 1.

\section{PROCEDURE}

This experiment consisted of two consecutive phases. In the first phase, rats were tested with an interruption procedure under the same visual conditions as they experienced in Experiment 1. In the second phase, the lighting conditions were reversed. Rats trained previously in darkness were trained in transparent tunnels under white light, and rats trained previously in transparent tunnels under white light were trained in darkness.

PHASE 1: UNCHANGED LIGHTING CONDITIONS

Prior to each trial, the entrance to four arms, arbitrarily chosen for each test and for each ani-

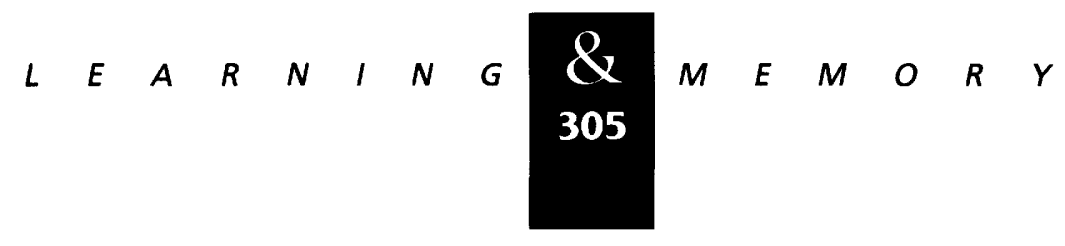


mal, was blocked by Plexiglas sliding doors. The starting procedure was the same as in Experiment 1. The rat was allowed to make choices until the four unblocked arms had been visited. The rat was then interrupted, removed from the maze, and placed in a bucket, following which the experimenter opened the four blocked arms. At this same time, four arms of the maze could also be permuted in order to dissociate olfactory and spatial cues (see Condition 2 below). The rat was then returned to the center of the maze and allowed to choose among all eight arms until the four previously blocked arms had been visited. Two training conditions, an unchanged maze and a premuted maze, were used successively.

\section{CONDITION 1: UNCHANGED MAZE}

In the first six trials, the maze was unchanged during the interruption, that is, each maze arm stayed in the same location. Thus, the olfactory and the spatial cues were coherent, and rats could rely either on the spatial or on the olfactory information to choose the four previously blocked arms.

CONDITION 2: PERMUTATION OF MAZE ARMS

During the next six trials, four arms of the maze were permuted during the interruption to dissociate olfactory and spatial information. Arms were baited according to the specific olfactory cues marking the arms that were not visited before the interruption. Thus, only the olfactory cues were predictive of the presence of the reinforcement (Fig. 4). The olfactory and the spatial cues were dissociated in four of the arms, but four arms maintained coherent olfactory and spatial information. There were thus four different pairs of arms, according to the predictability of the presence of the reinforcement by the different cues: Olf + Spat + (baited arms, both the olfactory and the spatial cues predicted the presence of the reinforcement); Olf + Spat - (baited arms, the olfactory cues predicted the presence of the reinforcement, whereas the spatial cues predicted the absence of the reinforcement); Olf-Spat + (unbaited arms, the olfactory cues predicted the absence of the reinforcement, whereas the spatial cues predicted the presence of the reinforcement); and Olf-Spat- (unbaited arms, both types of cues predicted the absence of the reinforcement).

PHASE 2: REVERSED LIGHTING CONDITIONS

Rats trained in darkness or under white light in transparent tunnels were then tested under the reversed lighting condition. The rats trained previously in transparent tunnels under white light were now tested in darkness [darkness + odor (wl) $(n=8)$ ] whereas those trained previously in darkness were now tested in transparent tunnels under white light [transparent + odor $(d)$ $(n=9)]$.

Figure 4: Schematic representation of the maze arms with the interruption procedure. (1) The baited arms; the arrangement of the baited and unbaited arms represents one of the different possibilities used during testing. (a) Before the interruption, four arms were open and baited (arms 1,2,4,6), whereas four arms were blocked (arms 3,5,7,8). (b) After the interruption, the eight arms

a Before interruption

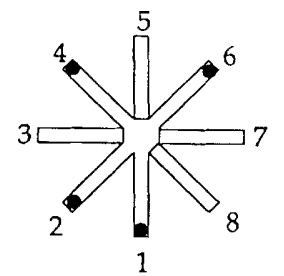

b

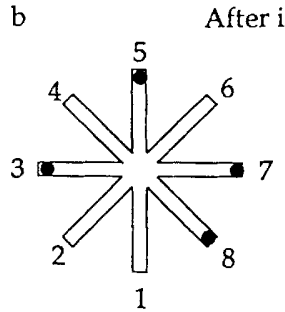

Unchanged maze
After interruption

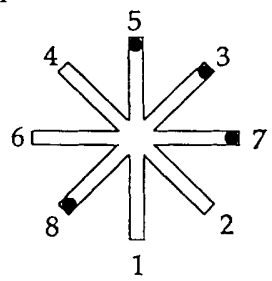

Permutation of maze arms were open and the four previously blocked arms (arms 3,5,7,8) were baited. In the unchanged maze condition, the olfactory and the spatial information were coherent. In the permuted maze arms condition, two pairs of arms were permuted ( 8 with 2 and 3 with 6), which dissociated olfactory and spatial information. Four different types of arms were defined according to the predictability of the presence of the reinforcement by the different type of cues: Olf + Spat $+($ arms $5,7)$ : baited arms, both the olfactory and the spatial cues predict the presence of the reinforcement; Olf + Spat $-($ arms 3,8 ): baited arms, the olfactory cues predict the presence of the reinforcement, whereas the spatial cues predict the absence of the reinforcement; Olf-Spat + (arms 2,6): unbaited arms, the olfactory cues predict the absence of the reinforcement, whereas the spatial cues predict the presence of the reinforcement; Olf-Spat-(arms 1 and 4): unbaited arms, both types of cues predict the absence of the reinforcement.

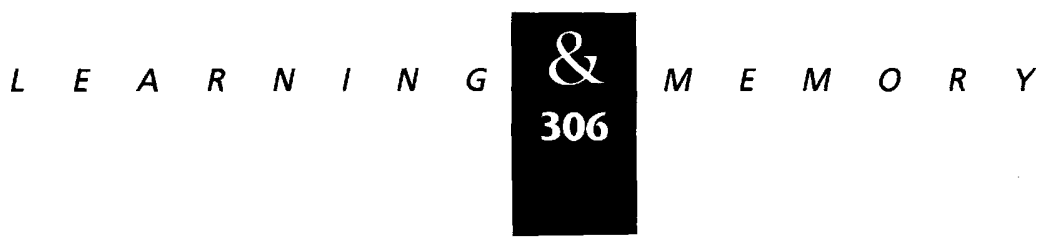


The interruption procedure and the two conditions of training (unchanged maze and permutation of the maze's arms) were the same as in the unchanged lighting conditions (Phase 1). The presence of the reinforcement was, as before, predicted by the olfactory cues.

The performance of the animals is described with regard to the number of errors (entries into already visited arms) and to the number of each arm type visited in the first four choices after the interruption (i.e., Olf + Spat + , Olf + Spat - , Olf - Spat + , and Olf - Spat - ).

\section{Results}

\section{PHASE 1: UNCHANGED LIGHTING CONDITIONS}

A two-way anova with repeated measures indicated a significant difference between groups in the number of errors (Fig. 5a) $[F(2,21)=26.31$, $P=0.0001]$, as well as a difference between maze conditions after the interruption $[F(1,21)=75.02$, $P=0.0001]$, with a significant interaction between both factors $[F(2,21)=38.8, P=0.0001]$.

When the maze remained unchanged during the interruption, results were coherent with those obtained in experiment 1 . Rats tested with translucent tunnels made more errors than those tested either in transparent tunnels or in darkness $[F(2,21)=8.75, P=0.002$, translucent + odor $>$ transparent + odor $=$ darkness + odor $)$.

The permutation of the maze arms during the interruption did not induce any disruption of the performance of the rats trained in darkness $[F(1,8)=2.78, P=$ ns $]$, whereas the rats of the two other groups made more errors when the arms were permuted and the olfactory cues only were predictive of the presence of the reinforcement [transparent + odor: $F(1,7)=101.24, P=0.0001$; translucent + odor: $F(1,6)=18.31, P=0.006$ ].

Thus, when rats had to rely on the olfactory cues, the group tested in darkness made fewer errors than those tested under white light either in transparent or translucent tunnels $[F(2,21)=44.99$, $P=0.0001$, darkness + odor $<$ transparent + odor $=$ translucent + odor $]$.

The analysis of the arm types chosen in the first four choices after the interruption, following the dissociation of the olfactory and the spatial cues (Fig. 5b) shows different patterns according to the experimental condition (arm types: $F(3,63)=116.92, P=0.0001$; interaction between arm type and condition: $F(6,63)=39.88$, $P=0.0001)$. Rats trained in darkness chose preferentially the two arm types in which the olfactory cues predicted the presence of the reinforcement $[F(3,24)=136.88, P=0.0001$, Olf + Spat + $=$ Olf + Spat $->$ Olf - Spat $+=$ Olf - Spat - . Rats trained in translucent tunnels chose more often the
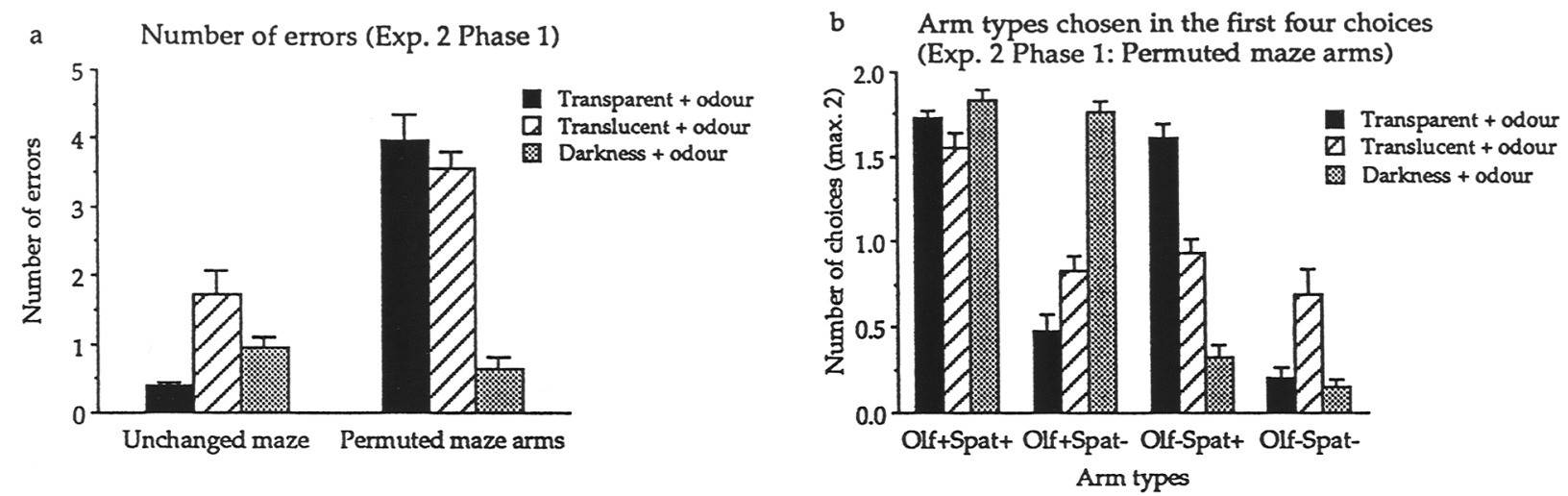

Figure 5: (a) Mean number of errors in the two maze conditions (unchanged maze and permuted maze arms) under unchanged lighting conditions (Phase 1 of Experiment 2). (b) Arm types chosen in the first four choices after the interruption in the permuted maze arms condition under unchanged lighting conditions (Phase 1 of Experiment 2). Olf + Spat + : baited arms, both the olfactory and the spatial cues predict the presence of the reinforcement; Olf + Spat - : baited arms, the olfactory cues predict the presence of the reinforcement, whereas the spatial cues predict the absence of the reinforcement; Olf -Spat + : unbaited arms, the olfactory cues predict the absence of the reinforcement, whereas the spatial cues predict the presence of the reinforcement; Olf - Spat - : unbaited arms, both types of cues predict the absence of the reinforcement.

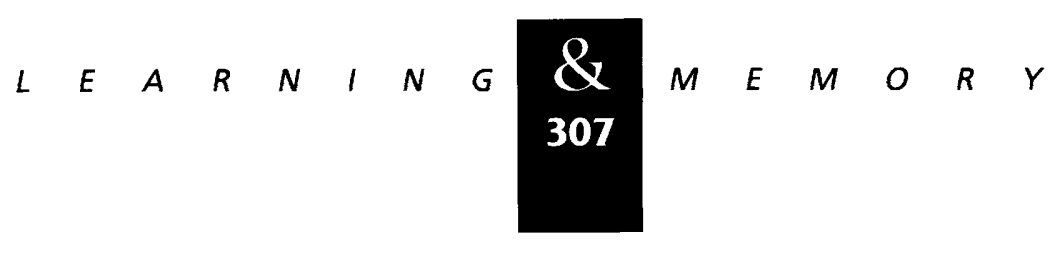


arms where both types of cues were predictive of the presence of the reinforcement, as compared with the other types of arms, which were all chosen with a similar frequency regardless of the types of cues $[F(3,18)=9.46, P=0.0006$, Olf + Spat $+>$ Olf + Spat $-=$ Olf - Spat $+=$ Olf - Spat -$]$.

\section{PHASE 2: REVERSED LIGHTING CONDITIONS}

A two-way ANOVA with repeated measures conducted on the number of errors (Fig. 6a) indicated no difference between groups $[F(1,15)=0.17, P=\mathrm{ns}]$, a significant difference between maze conditions after the interruption $[F(1,15)=39.89, P=0.0001]$ and no interaction between the two factors $[F(1,15)=1.79, P=n s]$. Both groups of rats made more errors when the reinforcement was predicted only by the olfactory cues, whatever the lighting conditions.

When the olfactory and the spatial information were dissociated and the reinforcement was predicted only by the olfactory cues, rats of both groups tended to use the same strategy to choose arms after the interruption (Fig. 6b). They entered preferentially the arms in which both the olfactory and the spatial information predicted the presence of the reinforcement and neglected those where both predicted the absence of reinforcement. The arms in which the olfactory and the spatial information conflicted were chosen with an intermediate frequency [arm types: $F(3,45)=55.13$, $P=0.0001$; interaction: $F(3,45)=2.58, P=\mathrm{ns}$; Olf + Spat $+>$ Olf + Spat $-=$ Olf - Spat $+>$ Olf - Spat -$]$.

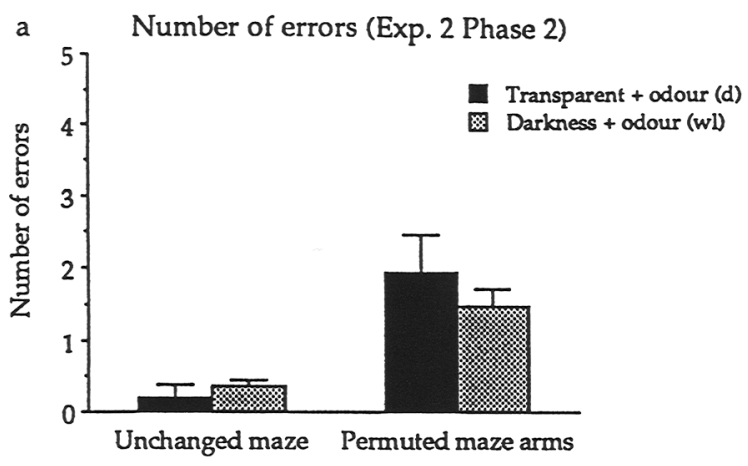

\section{Discussion}

The results of Phase 1 (unchanged lighting conditions) are coherent with those obtained in Experiment 1. The interruption procedure did not reduce performance in any condition, and rats remained as efficient as they were without interruption (Fig. 5a). However, when the arms were permuted during the interruption, only rats trained in darkness were able to use the supplementary olfactory cues to perform the task with a high degree of accuracy (Fig. 5b). In this condition they were able to use the supplementary olfactory cues even though their configuration was disrupted during each daily interruption. This demonstrates that in darkness rats can use the olfactory cues as a list of independent items marking each arm and they do not need the configuration of the olfactory cues to remain stable to solve the task. This contrasts with visual cues, which have been shown to be used in a configurational manner rather than as a list of items (Suzuki et al. 1980). It has already been shown that rats can solve a radial maze task by using intramaze cues, the locations of which can either remain stable or vary throughout training. However previous experiments were usually conducted with multimodal cues (i.e., the tactile and visual cues inserted along the arms must also have a specific odor) to enhance arm discrimination (e.g., Kraemer et al. 1983; M'Harzi and Jarrard 1992).

In Phase 2 (reversed lighting conditions), rats from both groups relied on the spatial and on the olfactory information to perform the task. They

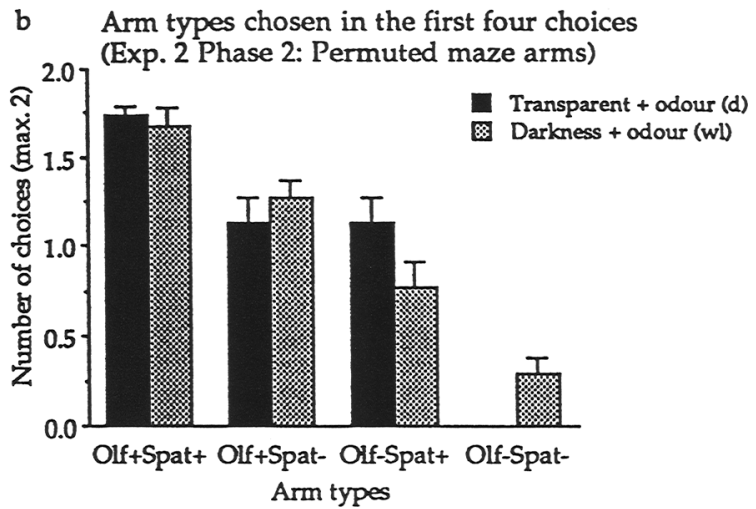

Figure 6: (a) Mean number of errors in the two maze conditions (unchanged maze and permuted maze arms) under reversed lighting conditions (Phase 2 of Experiment 2). (b) Arm types chosen in the first four choices in the permuted maze arms condition under reversed lighting conditions (Phase 2 of Experiment 2). The different arm types are the same as described in Phase 1 (Fig. 4).

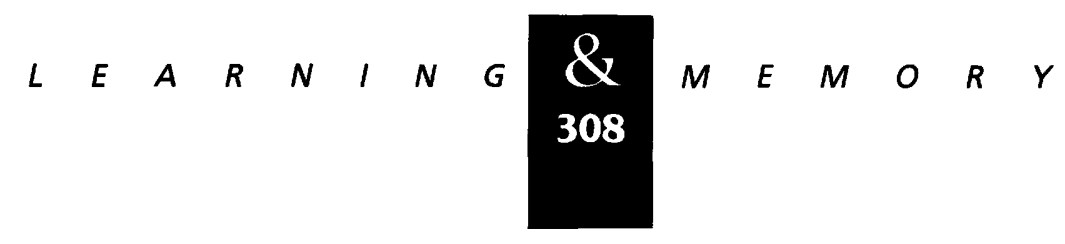


considered both types of cues to be predictive of the presence of the reinforcement, whether they had access to visuospatial cues or not (Fig. 6b). These results contrast with those obtained in the first part of the experiment (unchanged lighting conditions). They indicate that previous training in darkness enhanced the reliance on the olfactory cues when rats were further tested under white light, whereas pretraining in transparent tunnels enhanced the reliance on the spatial information when rats were tested in darkness. These results are particularly interesting as they show that rats trained previously in the light but tested in darkness still rely on spatial information that was provided previously by visual cues, even though these cues are no longer perceptible. Experiments on the firing of place cells in the hippocampus (Quirk et al. 1990; Sharp et al. 1990) have shown that some cells continue to fire even though visual cues are suppressed while the rat is still in the apparatus. These cells do not respond, however, when the rat is introduced into the apparatus in darkness; whereas our experiments suggest a more resistant memory of this reference.

Information about absolute space was unlikely to be provided by uncontrolled external auditory or magnetic cues. The influence of auditory cues had been suggested (Honzik 1936) and can't be ruled out totally, but the few attempts to demonstrate their role in radial maze experiments has led to negative results (Zoladek and Roberts 1978; Schaeppi et al. 1991). The potential of different species of mammals to use magnetic information is controversial (e.g., Mather and Baker 1981; Madden and Phillips 1987). Burda et al. (1990) have shown that, in an experimental arena, mole rats (Cryptomys bottentotus) change their nest position according to a change in the magnetic north, but to our knowledge, no finding has ever demonstrated magnetic perception in the norway rat (Rattus norvegicus).

In contrast, the integration of the vesibular and kinesthetic information generated by the animal's own movements (path integration mechanism) might inform the rat about its position with respect to a given point of departure. Several experiments conducted in rats (Zoladek and Roberts 1978; Schaeppi et al. 1991; Ossenkopp and Hargreaves 1993) and hamsters (Etienne et al. 1994) have shown the role of vestibular and kinesthetic information in solving maze problems. Another experiment conducted in our laboratory (Lavenex 1995 ) showed the role of vestibular information in the eight-arm radial maze task. Rats were trained over a prolonged period in the absence of discriminative cues, with the same type of interruption procedure. Their performance was impaired if we disturbed their path integration mechanism by placing the rats on a slow rotating platform $\left(90^{\circ} \%\right.$ sec) during the interruption. Our experiment suggested that if adequate procedures were used to enable the anchoring of the spatial representation to a particular location, such as the bucket where the rat was waiting during the interruption, the rat could rely on the information derived from its vestibular and kinesthetic systems to determine its position, even if the availability of visuospatial cues was suppressed before the rat was placed in the apparatus.

The results of Experiment 2 established that rats can use the olfactory cues as a list of independent items marking each arm to solve the task in darkness. They indicate also that pretraining in darkness with olfactory cues could enhance the rat's subsequent reliance on these cues under white light.

\section{Experiment 3}

Experiment 1 demonstrated that rats were sensitive to the modification of the configuration of olfactory cues, suggesting that they remembered the relative position of each arm. Experiment 2 demonstrated that, in darkness, rats could use the olfactory cues as a list of items independently of their location. The present experiment was designed to determine whether rats can rely on the configuration of olfactory cues to solve the radial maze task. More specifically, we analyzed whether rats could discriminate an arm on the sole basis of its position relative to that of other scented arms.

Rats were trained in darkness in an eight-arm radial maze with supplementary olfactory cues. Only five arms received a distinct olfactory cue, whereas the three remaining arms received the same supplementary olfactory cue. Thus, rats could not use these cues as a list of independent items marking each arm to solve the task. The arms sharing the same olfactory cue could only be identified by their position relative to that of the other scented arms. Thus, rats had to rely on the configuration of the supplementary olfactory cues to solve the task. The maze arms with the same olfactory cue could be permuted to control for the

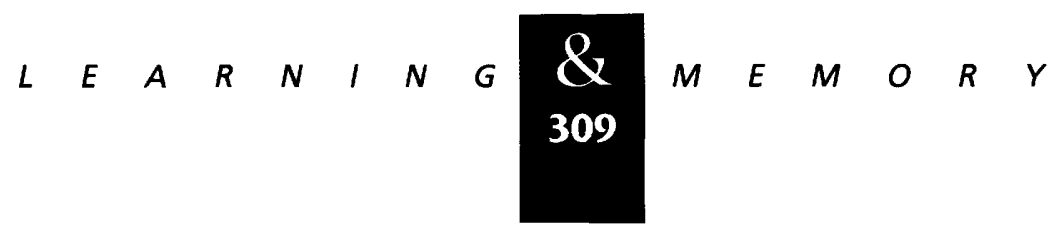


olfactory traces present on the maze floor. The maze could also be rotated to determine whether rats can rely on the configuration of the olfactory cues to solve the task independently of the external spatial frame of reference.

\section{Materials and Methods}

\section{SUBJECTS}

Eighteen male Long-Evans hooded rats were used as subjects. They were 3 to 5 months old at the beginning of the experiment and had no previous experience in any behavioral study. The housing and feeding conditions were the same as in the preceding experiments.

\section{APPARATUS}

The apparatus was the same as in the first two experiments. Supplementary olfactory cues were added to each arm, as described in Experiment 1. In this experiment, however, only five arms received a distinct olfactory cue (arms 1, 3, 4, 6, and 8 ), whereas the three remaining arms (arms 2, 5 , and 7) received the same supplementary olfactory cue.

\section{PROCEDURE}

STANDARD PROCEDURE

Rats were trained in darkness with a standard procedure without interruption similar to that used in Experiment 1. This phase lasted for 13 days with one trial per day.

One group of rats $(n=10)$ was trained with a stable position of the maze throughout training. Thus, the configuration of the supplementary olfactory cues was coherent with spatial information. Another group of rats $(n=8)$ was tested with the maze rotated between each daily session. Thus, the configuration of the olfactory cues was kept constant within the maze but was disconnected from the external spatial frame of reference.

The performance of the animals was described with regard to the number of errors (entries into previously visited arms) and to the strategy used to patrol the maze (angle separating two arms visited successively).

\section{INTERRUPTION PROCEDURE}

Rats trained previously with a stable maze position were then tested with an interruption procedure similar to that used in Experiment 2. Before the interruption, two arms with the same olfactory cue and two arms with a distinct olfactory cue were open. During the interruption, the maze could remain stable or could be rotated to dissociate the configuration of the olfactory cues from the external spatial frame of reference. The experimenter could also permute one of the two arms with the same olfactory cue visited before the interruption, with the third arm sharing the same olfactory cue, which had not been visited before the interruption. This manipulation was conducted to control for the olfactory traces left on the arms' floor, as when the arms were displaced, the tunnel and the arms' floor were moved. The information given by the olfactory traces present on the floor of the permuted arms was discordant from the information given by the configuration of the supplementary olfactory cues. After the interruption, the arms were baited according to the configuration of the supplementary olfactory cues. Thus, when the maze was manipulated, rats had to neglect the olfactory traces left on the maze floor, as well as the external spatial frame of reference. They had to rely solely on the configuration of the supplementary olfactory cues to solve the task. Three training conditions were used successively (Fig. 7).

\section{CONDITION 1: UNCHANGED MAZE}

In the first six trials, the maze was unchanged during the interruption. In this condition, rats had to solve the task while the configuration of the supplementary olfactory cues was coherent with the external spatial frame of reference and the olfactory traces present on the arms' floor.

There were four different arm types after the interruption, as defined by the presence of the reinforcement and the supplementary olfactory cue marking the arm. Two types of baited arms had not been visited before the interruption: one arm with the same olfactory cue common to three arms and three arms with distinct olfactory cues. Two types of unbaited arms had been visited before the interruption: two arms with the same olfactory cue and two arms with distinct olfactory cues.

$$
\begin{array}{lllllllllllllll}
L & E & A & R & N & I & N & G & \underset{310}{\mathbf{Z}} & M & E & M & O & R & Y
\end{array}
$$


a Before interruption

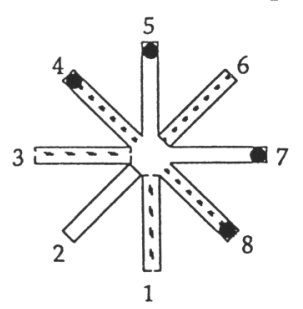

b

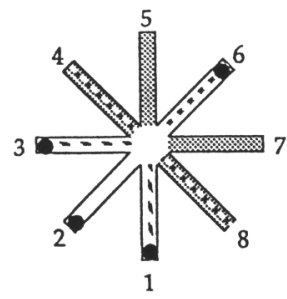

Unchanged maze
After interruption

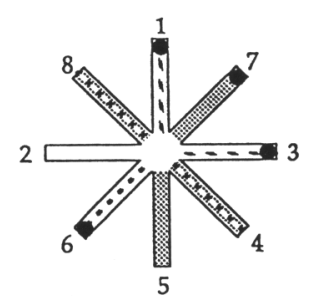

Arms' permutation + Maze's rotation

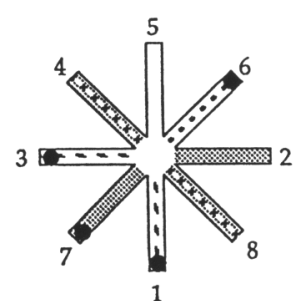

Arms' permutation

Figure 7: Schematic representation of the maze arms in the different training conditions with an interruption procedure in Experiment 3. The arrangement of the baited and unbaited arms represents one of the different possibilities used during testing. (1) The baited arms; the broken lines within the arms indicate the arms characterized by distinct olfactory cues. The arms with dashes represent arms visited before the interruption. (a) Before the interruption, two arms with distinct olfactory cues (arms 4,8) and two arms with the same olfactory cue (arms 5,7) were open and baited, whereas three arms with distinct olfactory cues (arms 1,3,6) and one arm with the same olfactory cue (arm 2) were blocked. (b) After the interruption, the eight arms were open and the arms were baited according to the configuration of the supplementary olfactory cues. In the unchanged maze condition, the olfactory and the spatial information were coherent. In the two permuted maze arms conditions (with or without maze rotation), two arms with the same olfactory cue were permuted (arm 7 previously visited and arm 2 previously blocked). Five different types of arms were defined according to the presence of the reinforcement, the supplementary olfactory cue characterizing the arm, and the olfactory traces present on the arms' floor: BSV (arm 7); baited arm, same olfactory cue common to three arms, previously visited (permuted); BDN (arms 1,3,6) baited arms, distinct olfactory cue for each arm, not visited; USN (arm 2): unbaited arm, same olfactory cue common to three arms, not visited (permuted) USV (arm 5): unbaited arm, same olfactory cue common to three arms, previously visited; UDV (arm 4,8): unbaited arms, distinct olfactory cue for each arm, previously visited.

\section{CONDITION 2: ARMS' PERMUTATION AND MAZE ROTATION}

In the next six trials, the maze was rotated and two arms with the same olfactory cue were permuted during the interruption. Rats had to rely on the configuration of the supplementary olfactory cues, which was no longer linked to the external spatial frame of reference. They had also to neglect the olfactory traces present on the floor of the permuted arms to find the baited arms. Thus, to discriminate between the arms with the same olfactory cue, rats had to remember their position relative to that of arms characterized by a distinct olfactory cue.

There were five different arm types as defined by the supplementary olfactory cue characterizing the arm, the presence of the reinforcement, and the olfactory traces left on the floor of the arms sharing the same olfactory cue. The olfactory traces on the floor of the arm characterized by distinct olfactory cues were always associated with the supplementary olfactory cues. There were thus only two different arm types with distinct olfactory cues: three baited arms with distinct olfactory cues, not visited before the interruption (BDN) and two unbaited arms with distinct olfactory cues, visited before the interruption
(UDV). The olfactory traces on the floor of the three arms sharing the same olfactory cues were incoherent with the configuration of the olfactory cues for two of the arms. There were thus three different arm types: one baited arm with the same olfactory cue, visited before the interruption and permuted (BSV); one unbaited arm with the same olfactory cue not visited before the interruption and permuted (USN); and one unbaited arm with the same olfactory cue, visited before the interruption and not permuted (USV).

CONDITION 3: ARMS' PERMUTATION

During the last six trials, two arms with the same olfactory cue were permuted, but the maze was not rotated. Rats had to rely on the configuration of the supplementary olfactory cues, which was linked to the external spatial frame of reference, to find the baited arms. To discriminate between the arms with the same olfactory cue, rats had to remember their position relative to that of arms characterized by a distinct olfactory cue and to neglect the olfactory traces present on the arm's floor. The different types of arms were the same as in the second phase of the experiment (arms' permutation and maze rotation), as defined by the

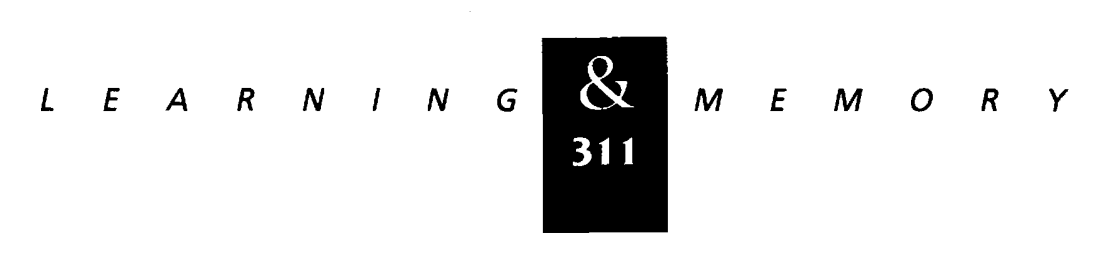


olfactory cue characterizing the arm, the presence of the reinforcement, and the olfactory traces present on the arms' floor (i.e., BDN, UDV, BSV, USN, USV).

The performance of the animals was described with regard to the number of errors (entries into previously visited arms) and to the arm types visited in the first four choices after the interruption.

\section{Results}

\section{STANDARD PROCEDURE}

The number of errors made during the first phase of training with a standard procedure without interruption (Fig. 8a) revealed no difference between groups $[F(1,16)=1.3, P=n s]$, and a decrease throughout sessions $[F(3,48)=5.9$, $P=0.0016]$ without interaction between groups and sessions $[F(3,48)=0.51, P=\mathrm{ns}]$.

However, the strategy used by rats to patrol the maze was different when the maze was stable or rotated between days (Fig $8 \mathrm{~b}$ ). In the last block of trials, the analysis of the number of adjacent arms visited successively revealed that the animals trained with a stable maze chained more frequently arms separated by $45^{\circ}$ than did rats trained with a daily rotation of the maze $[F(1,16)=7.01$, $P=0.0175]$. A detailed analysis of the other angles between arms successively visited was not presented as it gave redundant information.

\section{INTERRUPTION PROCEDURE}

The number of errors made in the different phases conducted with an interruption procedure

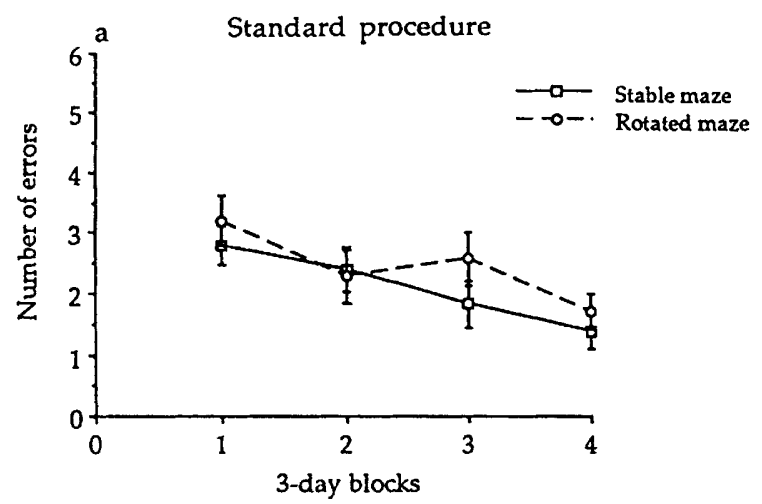

is represented in Figure 9a. It revealed that the arms' permutation + maze rotation procedure induced a large increase in the number of errors, whereas the number of errors made after arms' permutation without rotation of the maze was only slightly higher than that made in the unchanged maze condition $[F(2,19)=54.59$, $P=0.0001$, unchanged maze $<$ arms' permutation $<$ arms' permutation + maze rotation].

When rats were tested in an unchanged maze after the interruption, they entered preferentially the baited arms and made the same number of reentries into the arms sharing the same olfactory cue as in the arms characterized by distinct olfactory cues $[F(3,27)=62.27, P=0.0001$; baited, same cue $=$ baited, distinct cue $>$ unbaited, same cue $=$ unbaited, distinct cue] (not shown). The analysis of the arm types chosen in the first four choices after the interruption in the last two conditions (arms' permutation + maze rotation and arms' permutation) is shown in Figure $9 \mathrm{~b}$. When the arms were permuted and the maze was rotated during the interruption, rats chose preferentially the arms that did not bear olfactory traces left on the floor before the interruption, even though the configuration of the olfactory cues predicted the absence of the reinforcement $[F(4,36)=8.48$, $P=0.0001, \mathrm{BDN}=\mathrm{USN}>\mathrm{BSV}=\mathrm{USV}=\mathrm{UDV})$. When the arms were permuted but the maze was not rotated, rats entered preferentially the arms characterized by distinct olfactory cues, which had not been visited before the interruption (BDN). They neglected the unbaited arms that had been visited before the interruption and had not been permuted, whether they were characterized by distinct olfactory cues (UDV) or by the same olfac-

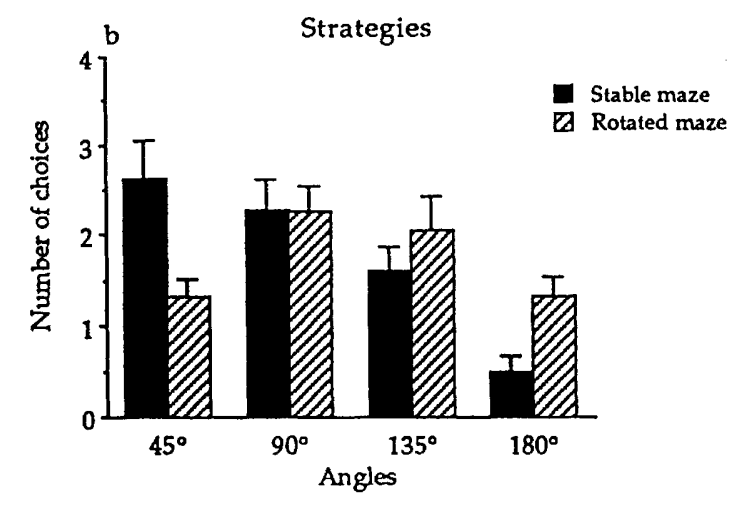

Figure 8: Number of errors per block of 3 days in the first phase without the interruption of Experiment 3. (b) Mean number of different angles separating two successively visited arms in the first eight choices during the last block of trials (block 4).

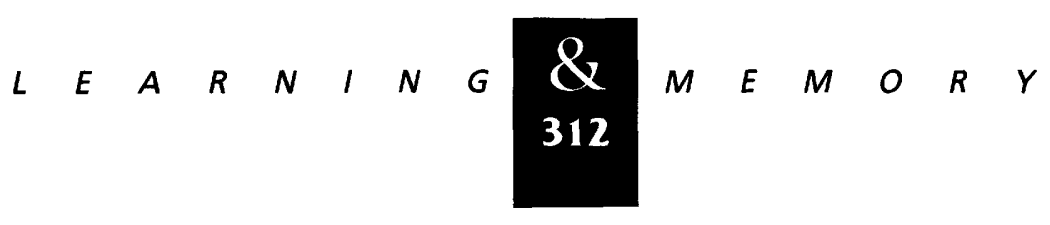



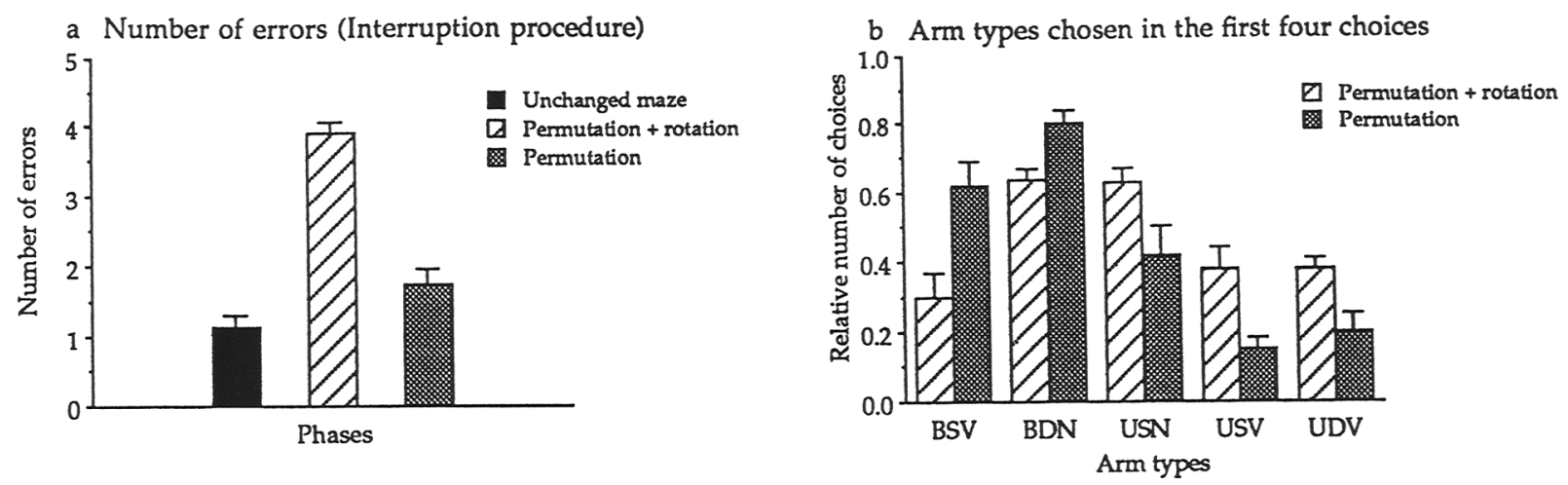

Figure 9: (a) Mean number of errors in the three different conditions of training (unchanged maze; permutation of maze arms and rotation of the maze; permutation of maze arms). (b) Arm types chosen in the first four choices in the two conditions with permuted maze arms (permutation of maze arms and rotation of the maze; permutation of maze arms). The number of choices of different arm types is given as the relative number of choices calculated according to the number of arms of each type. Arm types: (BSV) baited arm, same olfactory cue common to three arms, previously visited (permuted); (BDN) baited arms, distinct olfactory cue for each arm, not visited; (USN) unbaited arm, same olfactory cue common to three arms, not visited (permuted); (USV) unbaited arm, same olfactory cue common to three arms previously visited; (UDV) unbaited arms, distinct olfactory cue for each arm, previously visited.

tory cue common to three arms (USV). The two arms that were permuted and for which the configuration of the supplementary olfactory cues and the olfactory traces present on the floor gave contradictory information were chosen with an intermediate frequency $[F(4,36)=19.36, P=0.0001$, $\mathrm{BDN}>\mathrm{BSV}=\mathrm{USN}>\mathrm{USV}=\mathrm{UDV})$.

\section{Discussion}

During the first phase of training without interruption, there was no difference in arm choice accuracy when the configuration of the olfactory cues was linked to the spatial frame of reference or was dissociated from it by a daily rotation (Fig. 8a). However, the strategy of patrolling the maze, that is, the angle separating two arms visited successively, was different in both conditions (Fig. $8 b$ ). The rats trained with a fixed maze tended to patrol the maze like those trained in transparent tunnels under white light or in darkness with eight distinct supplementary olfactory cues marking each arm (Experiment 1); whereas rats trained with a daily rotated maze were similar to those trained in darkness in the absence of supplementary olfactory cues (Experiment 1). This indicates that the coherence between the olfactory cues and the external spatial frame of reference and not only the presence of discriminative cues influences the strategy used to patrol the maze.

During the interruption procedure, rats had no problem in solving the task when the maze was unchanged; that is, the configuration of the supplementary olfactory cues, the olfactory traces left on the floor, and the spatial information were coherent (Fig. 9a). They did not commit more errors in the arms sharing the same supplementary olfactory cue than in the arms characterized by distinct olfactory cues. When the maze was rotated and some of the arms permuted, rats did not rely on the configuration of the supplementary olfactory cues. They entered preferentially the arms in which the olfactory traces present on the floor indicated that the arms had not been visited before the interruption (Fig. 9b). It's very unlikey that rats could have perceived differences between the three arms sharing the same olfactory cue, as the same amount of diluted aroma from a single stock was added to each arm. Moreover, when two of the arms sharing the same supplementary olfactory cue were merely permuted but the maze was not rotated and, thus, the configuration of the olfactory cues was coherent with the external spatial frame, the rats' performance was only slightly lower than when the maze was unchanged. Rats clearly discriminated the baited arms according to their configuration, as defined by spatial and olfactory information, even though their choices were also influenced by the uncontrolled olfactory traces left on the maze floor. This indicates that the configuration of the olfactory cues is considered only when it is coherent with spatial information.

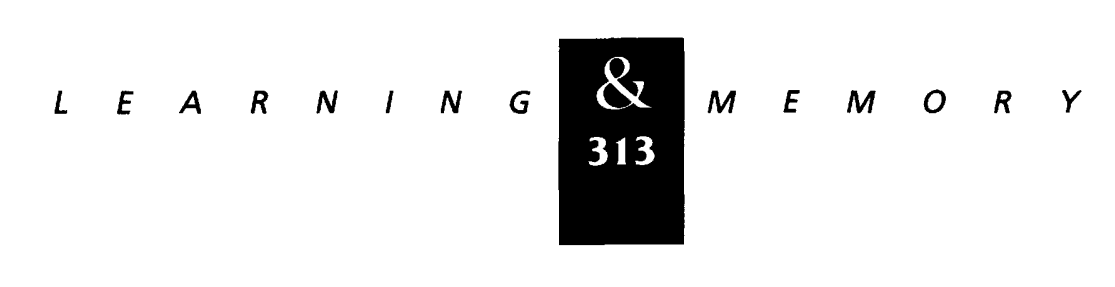


Experiment 3 demonstrated that rats were sensitive to the fact that olfactory cues were linked or dissociated from the external spatial frame of reference and that they did not rely on the mere configuration of the olfactory cues when it was disrupted from the spatial frame.

\section{Experiment 4}

Experiments 1 and 2 showed that rats did not rely on the supplementary olfactory cues for accurate arm choice in the absence of visuospatial cues when the maze was lighted (i.e., in translucent tunnels). The aim of this experiment was to verify whether the lack of efficiency in a translucent maze was specific to the olfactory information and to determine whether adequate cueing with intramaze visual cues could enable accurate arm choice in that condition.

\section{Materials and Methods}

\section{SUBJECTS}

Seven male Long-Evans hooded rats were used as subjects. They were 3 months old at the beginning of the experiment and had never been tested in any behavioral study. The housing and feeding conditions were the same as in the preceding experiments.

\section{APPARATUS}

The apparatus was the same as that used in the translucent conditions in Experiments 1 and 2 . Different visual cues, made of patches of black tape fixed to the sides and the ceiling of the tunnels, were added to each arm. They provided eight distinct visual patterns, that is, vertical stripes, small dots, horizontal stripes, etc., marking each arm individually. The availability of distant visuospatial cues was restricted by the use of translucent tunnels.

\section{PROCEDURE}

\section{STANDARD PROCEDURE}

Rats were first trained for 13 days with a procedure without interruption similar to that used in Experiment 1.

The performance was described in terms of the number of errors and was compared with the performance of the rats trained under white light in translucent or transparent tunnels in Experiment 1.

\section{INTERRUPTION PROCEDURE}

Rats were then tested with an interruption procedure similar to that used in Experiment 2. The maze remained unchanged during the interruption. The performance was described in terms of the number of errors and was compared with the performance of rats trained with supplementary olfactory cues under white light in translucent or in transparent tunnels in Phase 1 of Experiment 2.

\section{Results}

\section{STANDARD PROCEDURE}

The number of errors made during this first phase of training is shown in Figure 10a, together with the number of errors made by the animals trained in translucent tunnels or in transparent tunnels in Experiment 1. A one-way ANOVA conducted on the last block of trials revealed a significant difference between groups $[F(2,39)=4.41$, $P=0.0188$, translucent + visual $=$ transparent $<$ translucent). The performance of rats trained with translucent tunnels plus visual cues was similar to the performance of rats trained with full access to visuospatial cues and was better than that of rats trained in translucent tunnels with or without supplementary olfactory cues.

\section{INTERRUPTION PROCEDURE}

The number of errors made when the rats had to complete the maze after an interruption (Fig. $10 \mathrm{~b})$ reveals that the animals trained with translucent tunnels plus visual cues were not more accurate than the animals trained with translucent tunnels plus olfactory cues and made more errors than the animals trained in transparent tunnels $[F(2,29)=10.08, P=0.001$, transparent + odor $<$ translucent + visual $=$ translucent + odor $]$.

\section{Discussion}

This experiment shows that adequate cueing with visual cues, in contrast to olfactory cues, enables accurate arm choice in the translucent tunnels condition when there was no interruption

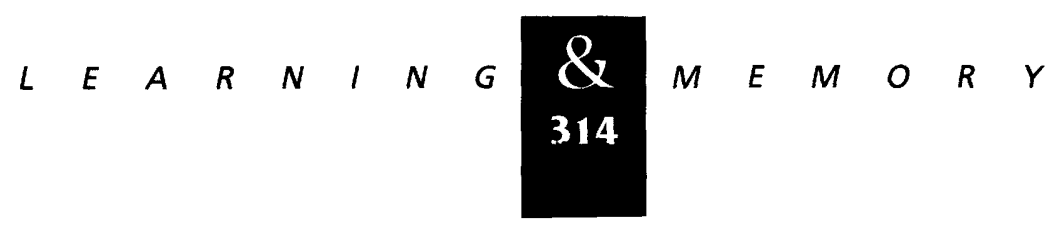



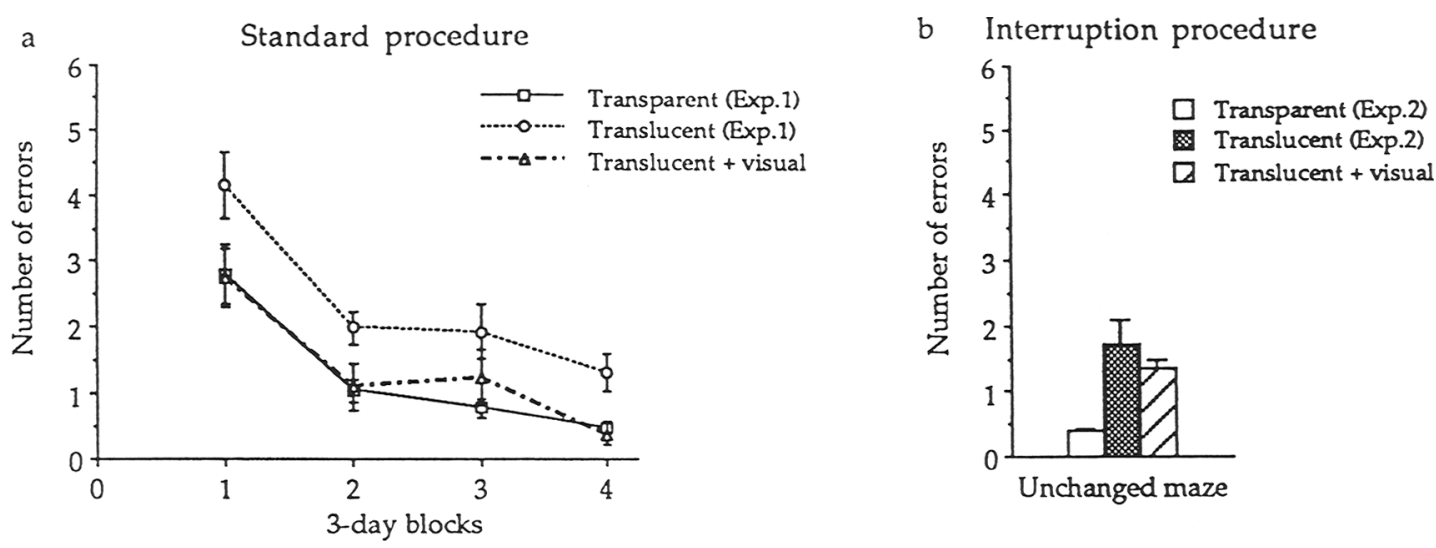

Figure 10: (a) Number of errors per block of 3 days in the first phase (without interruption) of Experiment 4 . (b) Mean number of different angles separating two successively visited arms in the first eight choices during the last block of trials (block 4).

(Fig. 10a). This demonstrates that the lack of efficiency in translucent tunnels with supplementary cues is specific to olfactory information. Nevertheless, the poor results obtained with the interruption procedure indicate that the use of intramaze visual cues is limited (Fig. 10b). These cues do not seem to enable the elaboration of a spatial representation similar to the one generated through the integration of the relationships between distant visual cues. In our experiment the rat could not perceive the visual cue characterizing one particular arm when it was in another arm. It is coherent with the study of Mazmanian and Roberts (1983) that showed that the restriction of the view of the environment, which prevented overlapping between views perceived from different arms, led to a decrease of performance when rats had to choose two arms out of four. It seems, thus, that rats need to have seen the different visual cues from different locations to establish a representation coding the spatial relations between these cues.

This experiment confirms that purely visual cues, in contrast to olfactory cues in darkness (Experiment 2), do not appear to be used as a list of independent items but are only used in a configurational manner to locate the arms (Suzuki et al. 1980). Moreover, in the present experiment, rats were not even able to correctly complete the maze after a short interruption.

\section{General Discussion}

The present study demonstrated that the olfactory cues enabled accurate arm choice in the radial arm maze on condition that the maze was not lighted, that is, only in darkness. In this condition the olfactory cues could be used as a list of independent items marking each arm, in contrast to visual cues. Rats, however, memorized the location of the olfactory cues whatever the lighting conditions. Nevertheless, they did not rely on the relative position of the olfactory cues, when these cues were dissociated from the external spatial frame of reference. These different points will be discussed successively.

\section{DIFFERENT MODES OF PROCESSING OF OLFACTORY INFORMATION ACCORDING TO LIGHTING CONDITIONS}

Previous studies have shown the predominance of the visuospatial cues over the olfactory cues when the two types of cues are in conflict (Olton and Collison 1979; Suzuki et al. 1980). It had been suggested, however, that even though visuospatial cues were the most relevant, olfactory cues might be used to direct arm choice if visuospatial cues are made less salient or even suppressed (Zoladek and Roberts 1978; Olton and Collison 1979). Our results contrast with this assumption, as the use of olfactory cues to direct arm choice did not depend on the availability of visuospatial cues but on the absence of light, even when the information provided by vision was null. Moreover, in previous studies the olfactory cues supposed to direct arm choice were the uncontrolled olfactory traces left on the floor of the maze. In our experiment the salience of the olfac-

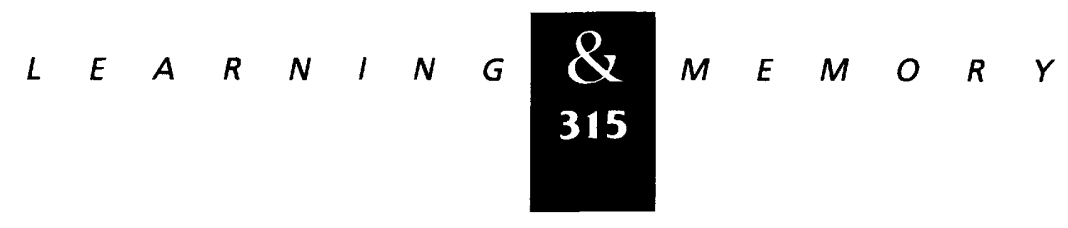


tory cues was greatly enhanced by the addition of supplementary olfactory cues, which were shown to be used for accurate arm choice in darkness. Experiment 4 showed that the lack of efficiency of the rats trained with supplementary olfactory cues in translucent tunnels was specific to the olfactory information, as visual cueing enabled maximal efficiency when the rats were allowed noninterrupted maze patrolling. Altogether, these results show that the presence of light limits the use of relevant olfactory cues, which are thus not used in an explicit manner.

Although the olfactory cues do not direct arm choice in the presence of light, they are much more efficient in directing arm choice in darkness than proximal visual cues are in translucent tunnels. The performances observed in the last block of training in Experiment 1 or in Experiment 2 show that the olfactory cues can be used as a list of independent items for accurate arm choice, in contrast to visual cues that can only be used in a configurational manner (Suzuki et al 1980; Mazmanian and Roberts 1983). Other types of cues can also be used as a list of independent items to solve the eight-arm radial maze. Independent multimodal visual and tactile cues could enable accurate arm choice (e.g., Kraemer et al. 1983; M'Harzi and Jarrard 1992). The use of the olfactory cues in darkness in our experiment supports previous findings showing the well-developed ability of rats to process olfactory information exhibited in nonspatial tasks (e.g., Staubli et al. 1987; Youngentob et al. 1990; Slotnick et al. 1991; Lu et al. 1993). Rats exhibit exceptionally rapid acquisition of operant discriminations when trained with odors (Nigrosh et al. 1975; Slotnick 1984) and when olfactory cues outperform visual cues in discrimination problems (Terrace 1963). Staubli et al. (1995) have trained rats to enter six reinforced arms marked by a specific odor in a 12-arm radial maze. Their efficacy $(\sim 35 \%$ of the trials without reentry) was noticeable but does not seem to reach that of a purely spatial task. Moreover, it was significantly reduced when a delay and a rotation of the maze was interposed after the third choice was made. In this condition it is not clear whether the delay or the rotation was the critical factor, as the rotation must require that the rat repeats visits to the same location to complete the task. Our results indicate that this type of task should be conducted in darkness, because in the absence of light the olfactory cues are used in an explicit manner and the rats are much more efficient in processing local olfactory than visual information in a radial maze.

The reactions elicited by the rearrangement of the olfactory cues in the absence of visuospatial cues and the different strategies used to patrol the maze in the presence or absence of supplementary olfactory cues reveal that rats attend to the olfactory cues even when the maze is lighted. Rats thus appear to have an implicit knowledge of the location of olfactory cues, which does not allow direct arm choice. The different strategies used to patrol the maze were clearly influenced by the presence of olfactory cues whatever the lighting conditions. Experiment 3 showed also that the strategy used to patrol the maze was influenced by the coherence between olfactory and spatial information. The rats of the three groups trained with eight distinct olfactory cues marking each arm in Experiment 1 and those trained with six distinct olfactory cues whose location was stable relative to the spatial frame in Experiment 3 exhibited the same type of maze patrolling usually observed under standard illumination in the presence of visuospatial cues (this paper; Suzuki et al. 1980; Yoerg and Kamil 1982; Innis and Macgillivray 1987). In contrast, the animals tested with a configuration of olfactory cues dissociated from spatial information in Experiment 3 tended to make larger angles between successive arms visited, very much like those tested in darkness without supplementary olfactory cues in Experiment 1. These results show that the strategies and not only the accuracy to solve the task should be considered to determine the influence of different types of cues in spatial tasks. If the accuracy to solve the task gives some indications on the cues that are used in an explicit manner, that is, to direct arm choice, the strategies and the reactions to changes in the environment reveal that some cues are processed in an implicit manner, as contextual cues, even though they do not enable accurate arm choice.

\section{OLFACTORY CUES IN A SPATIAL REPRESENTATION}

Previous findings have shown that hamsters memorize the location of olfactory cues associated with particular locations defined by their relationships with distant visual cues (Tomlinson and Johnston 1991). Our study shows that rats memorize the spatial configuration of the olfactory cues also in the absence of any visual cue, as long as the olfactory cues are coherent with the external spatial frame of reference.

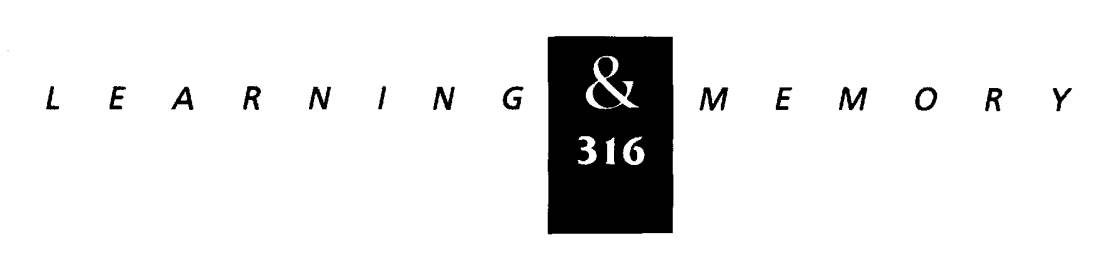


The reaction elicited by the rearrangement of the olfactory cues showed that rats formed a representation of the spatial configuration of the olfactory cues. The reaction to changes of the environment is commonly used to assess the kind of information encoded in a spatial representation (e.g., Poucet et al. 1986; Tomlinson and Johnston 1991). In our experiments rats reacted to the modification of the configuration of the olfactory cues in the absence of visuospatial cues, even when the maze was lighted. This shows that rats memorized the location of the olfactory cues, even though these cues did not enable accurate arm choice. The fact that the rats trained in the presence of visuospatial cues did not react to the modification of the configuration of the supplementary olfactory cues does not necessarily imply that they did not memorize the location of these cues. It is more likely that they did not show a measurable reaction to the modification of the location of the olfactory cues because the olfactory information was secondary relative to visuospatial information for an accurate performance. This suggests that rats memorize the location of the olfactory cues whatever the information provided by vision. This interpretation is coherent with the study of Tomlinson and Johnston (1991).

Even though olfactory cues are used to establish a spatial representation of the environment in the absence of visual information, this representation is linked to the external spatial frame of reference. When the olfactory cues could only be used as a configuration of cues for accurate arm choice, they had to remain stable relative to the spatial frame and were not used when they were disrupted from it. In the absence of visual cues, a spatial reference is unlikely to be provided by auditory cues (Zoladek and Roberts 1978; Schaeppi et al. 1991), even though their role cannot be totally ruled out. As stated previously, in the absence of any discriminative cue, the rats could still rely on the vestibular and kinesthetic information to orient themselves in the maze (Schaeppi et al. 1991; Ossenkopp and Hargreaves 1993), even with an interruption procedure after prolonged training (Lavenex 1995). Thus, in conditions in which the animals do not benefit from any discriminative cue, they can rely on the path integration mechanism to know their actual position with respect to a given point of departure (Mittelstaedt and Mittelstaedt 1980; Etienne 1992). One can thus infer that the location of the olfactory cues is linked to this reference framework.
This interpretation is supported by experiments on place cells in the hippocampus (Best and Thompson 1989), which showed that reliable information from the vestibular system was used to determine a cell's place-field location. When intramaze cues were inconsistent with existing reliable vestibular cues, then the place fields were not determined by intramaze cues. This is also coherent with the model proposed by McNaughton et al. (1995) on the central role that the vestibular information appears to play in establishing the fundamental directional reference framework that is used to construct cognitive representations of the environment based on visual cues. Our results show that olfactory cues are integrated in a spatial representation coding the topographical relations between these cues, as long as they are coherent with the external spatial frame of reference. It would be interesting to determine whether after prolonged training the spatial configuration of the olfactory cues could be used to determine the animal's location independently of any other spatial information. Knierim et al. (1995) have demonstrated that a visual cue could exert control on place and head direction cells independently of vestibular information on condition that rats could have established previously a consistent relationship between the cue and an internal sense of direction, maintained most likely by the vestibular system. This point will need further investigation.

In conclusion, the present study demonstrated that the use of olfactory cues to direct arm choice in the radial arm maze was critically dependent on the illumination conditions and implied two different modes of processing of olfactory information according to the presence or the absence of light. Clearly, olfactory cues were used in an explicit manner and enabled accurate arm choice only in the absence of light. Rats, however, had an implicit knowledge of the location of the olfactory cues and formed a representation of the spatial configuration of these cues whatever the lighting conditions. Nevertheless, rats did not memorize the spatial configuration of the olfactory cues per se but needed these cues to be linked to the external spatial frame of reference.

More generally, our results confirm that behavioral decisions such as leading to an arm choice are based on the relative weight of different categories of cues. This weight is based on a hierarchy for which the spatial framework appears to be a default reference. The weight of other cues is affected by their salience but can be modified by the

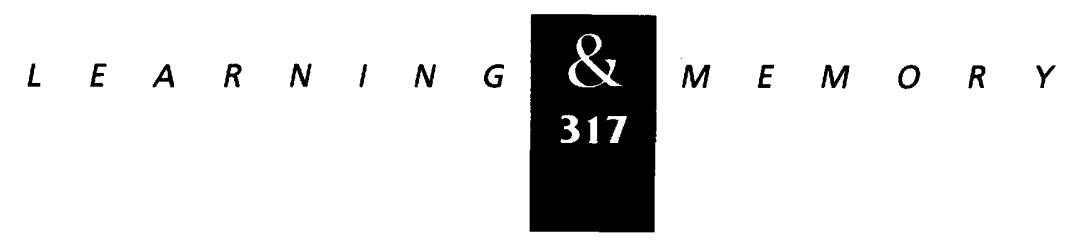


animal's past experience (see Experiment 2) or, for olfactory cues, by particular conditions such as the ambient light level. Whether the same hierarchy is valid in natural open-field situations has to be established but we have already found that the spatial reference framework still outweighs the local olfactory cues in an experimental open field (Lavenex and Schenk 1995). Whenever one type of cue is overshadowed by other prevalent cues, the direct or indirect effects of the overshadowed information on behavior need to be examined by considering the presence or the absence of the cues, the relative weight of all categories of cues, and the previous experience of the animal. It is also necessary to take into account different aspects of behavior to determine the effects of the overshadowed information, not only the overt behavior first considered.

\section{Acknowledgments}

This research was supported by a grant from the Fonds National de la Recherche Scientifique (31-39754.93).

The publication costs of this article were defrayed in part by payment of page charges. This article must therefore be hereby marked "advertisement" in accordance with 18 USC section 1734 solely to indicate this fact.

\section{References}

Alyan, S. and R. Jander. 1994. Short-range homing in the house mouse, Mus musculus: Stages in the learning of direction. Anim. Behav. 48: 285-298.

Baker, R.R. 1978. The evolutionary ecology of animal migration. Hodder and Stoughton, London, UK.

Best, P.J. and L.T. Thompson. 1989. Persistence, reticence, and opportunism of place-field activity in hippocampal neurons. Psychobiology 17: 236-246.

Burda, H., T. Marhold, R. Westenberger, R. Wiltschko, and W. Wiltschko. 1990. Magnetic compass orientation in the subterranean rodent Cryptomys hottentotus (Bathyergidae). Experientia 46: 528-530.

Buresova, O. and J. Bures. 1981. Role of olfactory cues in the radial maze performance of rats. Behav. Brain Res. 3: $405-409$.

Etienne, A.S. 1992. Navigation of a small mammal by dead reckoning and local cues. Psychol. Sci. 1: 48-51.

Etienne, A.S., E. Teroni, C. Hurni, and V. Portenier. 1990. The effect of a single light cue on homing behaviour of the golden hamster. Anim. Behav. 39: 17-41.

Etienne, A.S., S. Sitbon, C. Dahn-Hurni, and R. Maurer. 1994. Golden hamsters on the eight-arm maze in light and darkness: The role of dead reckoning. Q. J. Exp. Psychol. 47: 401-425.

Honzik, C.H. 1936. The sensory basis of maze learning in rats. Comp. Psychol. Monogr. 13: 1-113.

Innis, N.K. and M. Macgillivray. 1987. Radial maze performance under food and water deprivation. Behav. Proc. 15: $167-179$.

Knierim, J.J., H.S. Kudrimoti, and B.L. McNaughton. 1995. Place cells, head direction cells, and the learning of landmark stability. I. Neurosci. 15: 1648-1659.

Kraemer, P.J., M.E. Gilbert, and N.K. Innis. 1983. The influence of cue type and configuration upon radial-maze performance in the rat. Anim. Learn. Behav. 11: 373-380.

Lavenex P. and F. Schenk. 1995. Influence of local environmental olfactory cues on place learning in rats. Physiol. Behav. 58: 1059-1066.

Lu, X.-C.M., B.M. Slotnick, and A.M. Silberberg. 1993. Odor matching and odor memory in the rat. Physiol. Behav. 53: $795-804$.

M'Harzi, M. and L.E. Jarrard. 1992. Strategy selection in a task with spatial and nonspatial components: Effects of fimbria-fornix lesions in rats. Behav. Neural Biol. 58: $171-179$.

Madden, R.C. and J.B. Phillips. 1987. An attempt to demonstrate magnetic compass orientation in two secies of mammals. Anim. Learn. Behav. 15: 130-134.

Mather, J.G. and R.R. Baker. 1981. Magnetic sense of direction in woodmice for route-based navigation. Nature 291: 152-155.

Mazmanian, D.S. and W.A. Roberts. 1983. Spatial memory in rats under restricted viewing conditions. Learn. Motiv. 14: 123-139.

McNaughton, B.L., J.J. Knierim, and M.A. Wilson. 1995. Vector encoding and the vestibular foundations of spatial cognition: Neurophysiological and computational mechanisms. In The cognitive neurosciences (ed. M.S. Gazzaniga), MIT Press, Cambridge, MA.

Mittelstaedt, M.-L. and H. Mittelstaedt. 1980. Homing by path integration in a mammal. Naturwissenschaften 67: $566-567$.

Nigrosh, B.J., B.M. Slotnick, and J.A. Nevin. 1975. Olfactory discrimination, reversal learning, and stimulus control in rats. J. Comp. Physiol. Psychol. 89: 285-294.

Olton, D.S. and R.J. Samuelson. 1976. Remembrance of places passed: Spatial memory in rats. J. Exp. Psychol.: Anim. Behav. Proces. 2: 97-116.

Olton, D.S. and C. Collison. 1979. Intramaze cues and

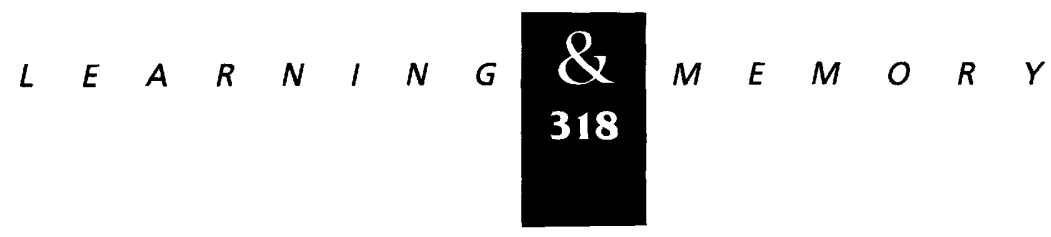


"odor trails" fail to direct choice behavior on an elevated maze. Anim. Learn. Behav. 7: 221-223.

Ossenkopp, K.-P. and E.L. Hargreaves. 1993. Spatial learning in an enclosed eight-arm redial maze in rats with sodium arsanilate-induced labyrinthectomies. Behav. Neural Biol. 59: 253-257.

Papi, F. 1990. Olfactory navigation in birds. Experientia 46: 352-363.

Poucet, B. 1993. Spatial cognitive maps in animals: New hypotheses on their structure and neural mechanisms. Psychol. Rev. 100: 163-182.

Poucet, B., N. Chapuis, M. Durup, and C. Thinus-Blanc. 1986. A study of exploratory behavior as an index of spatial knowledge in hamsters. Anim. Behav. 14: 93-100.

Quirk, G.J., R.U. Muller, and J.L. Kubie. 1990. The firing of hippocampal place cells in the dark depends on the rat's recent experience. J. Neurosci. 10: 2008-2017.

Schaeppi, U., G. Krinke, R.E. Fitzgerald, and W. Classen. 1991. Impaired tunnel-maze behavior in rats with sensory lesions: Vestibular and auditory systems. Neurotoxicology 12: $445-454$.

Schenk, F., B. Contant, and M.-C. Grobéty. 1990. Angle and directionality affect rat's organization of visit sequences and spatial learning in modular mazes. Learn. Motiv.

21: 164-189.

Sharp, P.E., J.L. Kubie, and R.U. Muller. 1990. Firing properties of hippocampal neurons in a visually symmetrical environment: Contributions of multiple sensory cues and mnemonic processes. I. Neurosci. 10: 3093-3105.

Slotnick, B.M. 1984. Olfactory stimulus control in the rat. Chem. Senses 9: 157-165.

Slotnick, B.M., A. Kufera, and A.M. Silberberg. 1991. Olfactory learning and odor memory in the rat. Physiol. Behav. 50: 555-561.

Staubli, U., D. Fraser, R. Faraday, and G. Lynch. 1987. Olfaction and the "data" memory system in rats. Behav. Neurosci. 101: 757-765.

Staubli, U., T.T. Le, and G. Lynch. 1995. Variants of olfactory memory and their dependencies on the hippocampal formation. J. Neurosci. 15: 1162-1171.

Still, A.W. and A.C. Macmillan. 1975. Location by odour and turn selection as two stages in the spontaneous alternation of rats. Anim. Behav. 23: 447-449.

Suzuki, S.W., G. Augerinos, and A.H. Black. 1980. Stimulus control of spatial behavior on the eight-arm maze in rats. Learn. Motiv. 11: 1-18.

Terrace, H.S. 1963. Discrimination learning with and without errors. J. Exp. Anal. Behav. 6: 1-27.
Tomlinson, W.T. and T.D. Johnston. 1991. Hamsters remember spatial information derived from olfactory cues. Anim. Learn. Behav. 19: 185-190.

Yoerg, S.I. and A.C. Kamil. 1982. Response strategies in the radial arm maze: Running around in circles. Anim. Learn. Behav. 10: 530-534.

Youngentob, S.L., L.M. Markert, M.M. Mozell, and D.E. Hornung. 1990. A method for establishing a five odorant identification confusion matrix task in rats. Physiol. Behav. 47: 1053-1059.

Zoladek, L. and W.A. Roberts. 1978. The sensory basis of spatial memory in the rat. Anim. Learn. Behav. 6: 77-81.

Received September 13, 1995; accepted in revised form March 8, 1996.

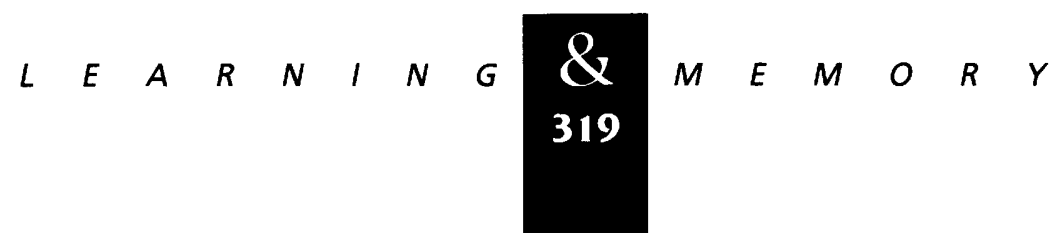




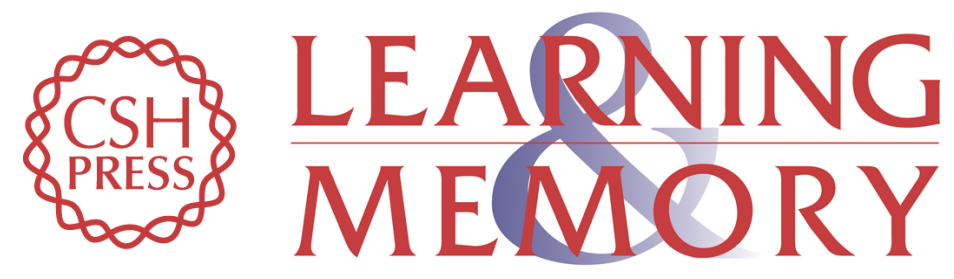

\section{Integration of olfactory information in a spatial representation enabling accurate arm choice in the radial arm maze.}

P Lavenex and F Schenk

Learn. Mem. 1996, 2:

Access the most recent version at doi:10.1101//m.2.6.299

References This article cites 40 articles, 4 of which can be accessed free at: http://learnmem.cshlp.org/content/2/6/299.full.html\#ref-list-1

License

Email Alerting Receive free email alerts when new articles cite this article - sign up in the box at the Service top right corner of the article or click here. 